Document downloaded from:

http://hdl.handle.net/10251/141972

This paper must be cited as:

Gao, P.; Climente Alarcón, A.; Sánchez-Dehesa Moreno-Cid, J.; Wu, L. (03-2). Theoretical study of platonic crystals with periodically structured $\mathrm{N}$-beam resonators. Journal of Applied Physics. 123(9). https://doi.org/10.1063/1.5009170

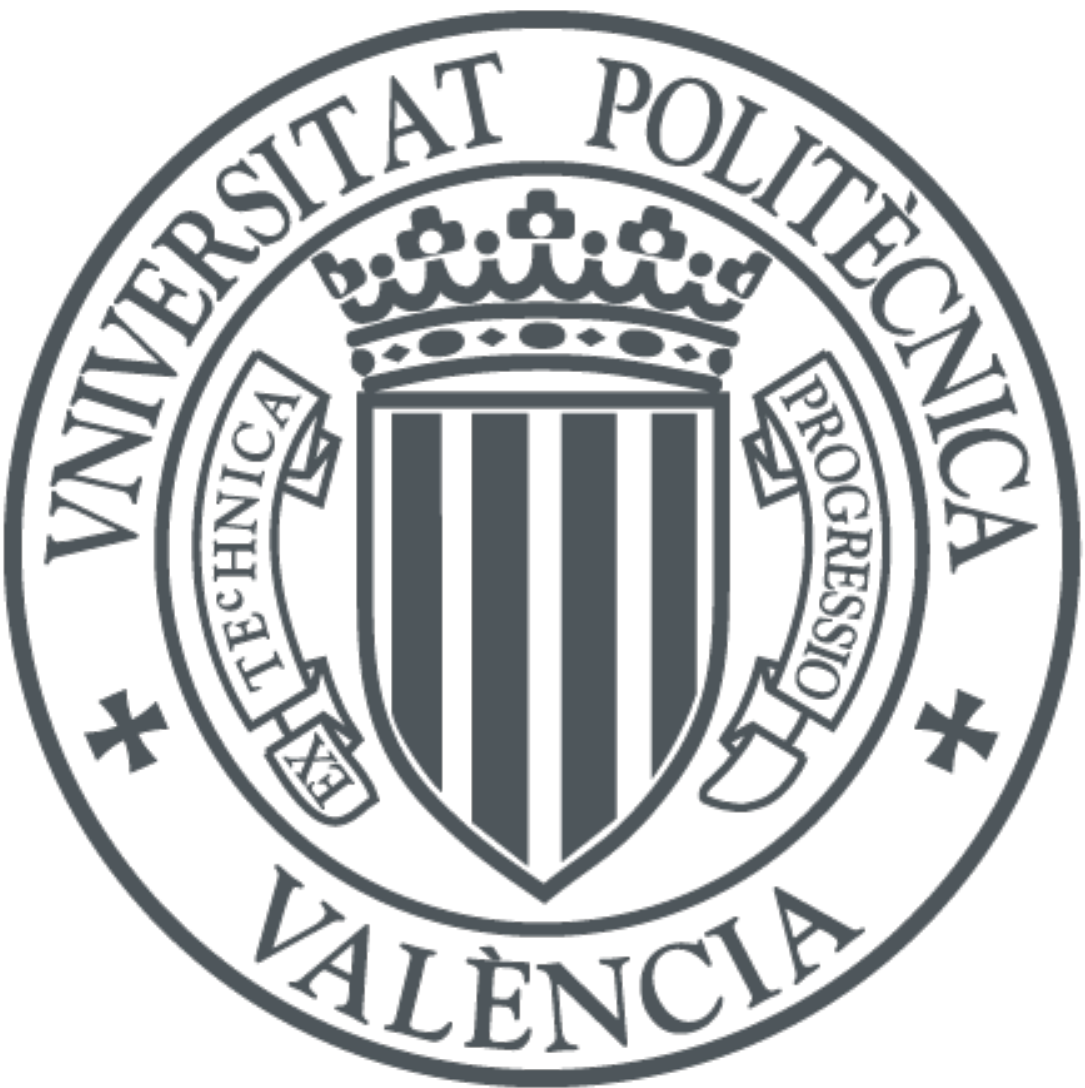

The final publication is available at

https://doi.org/10.1063/1.5009170

Copyright American Institute of Physics

Additional Information 


\section{Theoretical study of platonic crystals with periodically structured $N$-beam resonators}

Penglin Gao, ${ }^{1,2}$ Alfonso Climente, ${ }^{2}$ José Sánchez-Dehesa, ${ }^{2, a)}$ and Linzhi Wu', b)

1) Center for Composite Materials, Harbin Institute of Technology, Harbin 150001, China.

2) Wave Phenomena Group, Department of Electronic Engineering, Universitat Politècnica de València, Camino de vera s.n. (Building 7F), ES-46022 Valencia, Spain.

(Dated: 14 November 2017)

A multiple scattering theory is applied to study the properties of flexural waves propagating in a plate with periodically structured $N$-beam resonators. Each resonator consists of a circular hole containing an inner disk connected to background plate with $N$ rectangular beams. The Bloch theorem is employed to obtain the band structure of a two-dimensional lattice containing a single resonator per unit cell. Also, a numerical algorithm has been developed to get the transmittance through resonators slabs infinitely long in the direction perpendicular to the incident wave. For the numerical validation, a square lattice of 2-beam resonators has been comprehensively analyzed. Its band structure exhibits several flat bands, indicating the existence of local resonances embedded in the structure. Particularly, the one featured as the fundamental mode of the inner disk opens a bandgap at low frequencies. This mode has been fully described in terms of a simple spring-mass model. As a practical application of the results obtained, a homogenization approach has been employed to design a focusing lens for flexural waves, where the index gradient is obtained by adjusting the orientation of the resonators beams. Numerical experiments performed within the framework of a three-dimensional finite element method have been employed to discuss the accuracy of the models here described.

a)jsdehesa@upv.es

b)wlz@hit.edu.cn 


\section{INTRODUCTION}

As a kind of lightweight elastic structure, plates are of great significance in engineering. Many studies have been performed for elastic waves propagating in thin plates with periodically arranged microstructures owing to the novel wave propagation behaviors, like bandgaps, waveguiding and negative refraction. ${ }^{1,2}$ Additionally, the periodically structured plates provide a route to construct elastic materials with adjustable parameters which are not available in nature materials. ${ }^{3-6}$ At low frequencies, the wavelength is much larger than the lattice constant and, consequently, the plates with microstructures can be treated as if they were homogeneous. Homogenization theories have been developed to extract the effective parameters of these structured materials, usually called metamaterials. ${ }^{3,4}$ Gradient index lens ${ }^{7-10}$ and negative index lens $s^{4,5,11-13}$ are typical examples showing the bright future of phononic crystals and metamaterials. Focusing and negative refraction have attracted considerable attention in recent years because the former is of practical interest in signal detection and energy harvesting, ${ }^{14,15}$ while the latter has potential applications in super-resolution imaging. ${ }^{16}$

Bandgaps are one of the most intriguing features of periodic structures and they have led to a variety of applications, such as filtering, waveguiding and vibration control. ${ }^{1,2}$ Bragg scattering and local resonance are the two mechanisms usually employed to open bandgaps. ${ }^{17}$ In the former case, the underlying mechanism lies in the destructive interference among the multiple scattered waves so that the bandgaps fall into the frequency regions where the wavelength is comparable to the lattice constant. Quite differently, owing to the tunability of local resonances, the bandgaps in the latter case can be extended to low frequency regions, indicating a promising route to control noise and vibration in extremely low frequencies. ${ }^{18}$ Numerous works have been implemented to open resonance-type bandgaps for flexural waves by embedding spring-mass like resonators vibrating in the out-of-plane direction, such as binary locally resonant structures, ${ }^{19,20}$ pillars,${ }^{21-23}$ spring-masses ${ }^{24}$ and holes with inner structures. ${ }^{13,25}$ Among these resonant structures, the last one, also named as $N$-beam resonator in Ref. 26, deserves more attention because it is of easy manufacturing by using laser or waterjet cutting machines, which is crucial in practical applications.

In the past decades, several well-developed theories, such as the plane-wave expansion method, finite-difference time domain method, multiple scattering theory and finite ele- 
ment method, have been widely used to study the wave propagation behaviors for periodic lattices. ${ }^{17}$ Among these methods, finite element method dominates owing to its high efficiency in dealing with periodic structures with complex unit cells, which is the common case for resonant structures. In fact, among the aforementioned works concerning resonancetype bandgaps, only a few of them were carried out theoretically with plane-wave expansion method. ${ }^{19,20,24}$ The other works ${ }^{13,21-23,25}$ were performed with the help of the finite element method. Fortunately, the scattering of flexural waves from a single $N$-beam resonator has been solved analytically by Climente et $a l .{ }^{26}$ with the impedance matrix method, which makes it possible to study the $N$-beam resonator-based platonic crystals theoretically with the multiple scattering theory. ${ }^{27,28}$

This paper aims to investigate the properties of flexural waves propagating in a platonic crystal consisting on a thin plate containing a periodic lattice of $N$-beam resonators. It is organized as follows. After this introduction, Sec. II shows the model developed for calculating band structures and transmission spectrum by employing the multiple scattering theory, some useful but tedious developments are given in the Appendices. In Sec. III, some numerical simulations are shown to validate the present theory and to reveal the propagation characteristics of Bloch waves, especially the resonance-type bandgaps. Afterwards, we design a gradient index focusing lens by tuning the orientation of beams in the 2-beam resonators. Finally, the work is summarized in Sec. IV, which also gives an outlook of potential applications of the model here introduced.

\section{FLEXURAL WAVE SCATTERING BY PERIODICAL ARRAY OF $N$-BEAM RESONATORS}

This section presents the formulation of the multiple scattering theory applied to flexural waves propagating in a perforated thin plate. It begins with a preliminary knowledge about plates and beams, then the multiple scattering theory is applied to an array of $N$-beam resonators with arbitrary and periodic distributions. 


\section{A. Modeling of plates and beams}

If a plate is thin enough to meet Kirchhoff's assumptions, under a harmonic excitation, the transverse (out-of-plane) displacement $W$ is governed by the biharmonic equation as follows: 29

$$
\nabla^{4} W-k_{p}^{4} W=0
$$

where $k_{p}=\left(\rho h \omega^{2} / D\right)^{1 / 4}$ is the wave number with $\omega$ being the angular frequency; $D=$ $E h^{3} / 12\left(1-\nu^{2}\right)$ is the rigidity with $E, \nu$ and $\rho$ being the Young's modulus, Poisson's ratio and density of the plate, respectively. Moreover, $h$ is the thickness of the plate. The time harmonic factor $e^{-i \omega t}$ is implicit in all the formulation but will be omitted throughout the paper for simplicity. The solution of Eq. (1) is expressed in polar coordinates as

$$
W(r, \theta)=\sum_{q=-\infty}^{\infty}\left[A_{q}^{J} J_{q}\left(k_{p} r\right)+A_{q}^{I} I_{q}\left(k_{p} r\right)+B_{q}^{H} H_{q}\left(k_{p} r\right)+B_{q}^{K} K_{q}\left(k_{p} r\right)\right] e^{i q \theta},
$$

where $J_{q}(\cdot)$ is the Bessel function, $H_{q}(\cdot)$ is the Hankel function of first kind, and $I_{q}(\cdot), K_{q}(\cdot)$ are the modified Bessel functions. The column matrices $A_{q}=\left[A_{q}^{J} A_{q}^{I}\right]^{t}$ and $B_{q}=\left[B_{q}^{H} B_{q}^{K}\right]^{t}$ contain the expansion coefficients for the incoming and scattered waves, respectively.

The scatterers here considered are $N$-beam resonators, consisting of a circular hole (with radius $R_{2}$ ) containing a smaller concentric circular plate (with radius $R_{1}$ ) connected to the background plate with $N$ uniformly distributed rectangular beams (with width $b$ ). As an example, resonators with 2 beams are plotted in Figure 1, where labels I and II are used to denote the regions inside the inner plate and in the background plate, respectively. In this work, it is assumed that the resonators are fabricated by a waterjet cutting machine and all parts are made of the same material as the background plate.

From the physical point of view, the field in region II, $W=W^{i n c}+W^{s c a t}$, can be separated into two parts, in which the incoming field is

$$
W^{i n c}(r, \theta)=\sum_{q=-\infty}^{\infty} F_{q}^{J I}\left(k_{p} r, \theta\right) A_{q},
$$

and the scattered field is

$$
W^{\text {scat }}(r, \theta)=\sum_{q=-\infty}^{\infty} F_{q}^{H K}\left(k_{p} r, \theta\right) B_{q}
$$

where the matrix $F_{q}^{\Upsilon \Phi}(\xi, \eta)=\left[\Upsilon_{q}(\xi) \Phi_{q}(\xi)\right] e^{i q \eta}$ is defined for simplicity. Notice that $H_{q}(\cdot)$ and $K_{q}(\cdot)$ get infinite values at the origin point, therefore they have to be discarded and the 

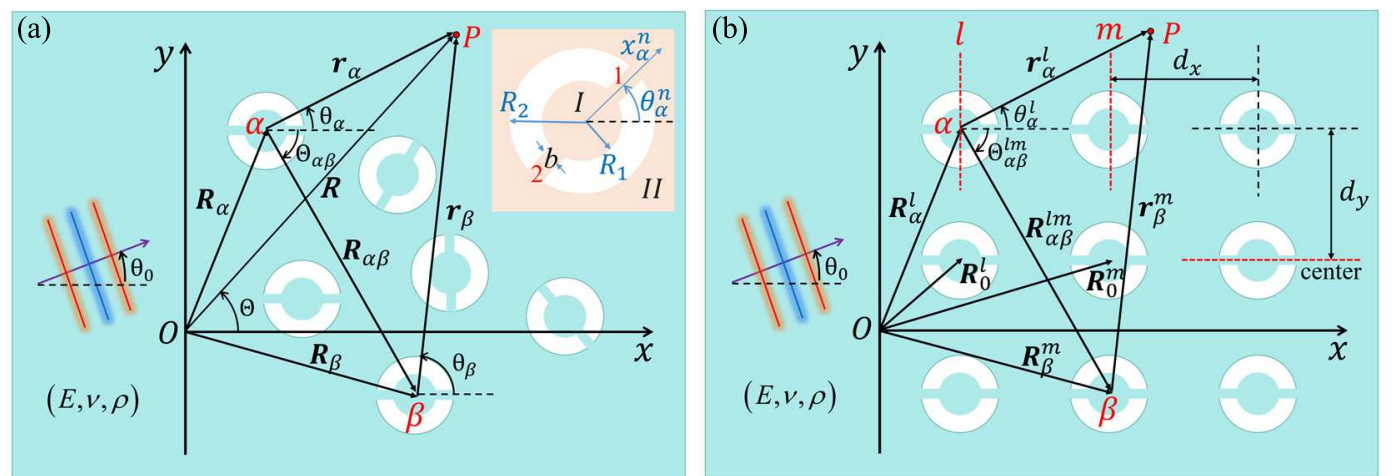

FIG. 1. (color on line) Definition of the systems of coordinates and variables employed in the expressions of the multiple scattering theory. (a) Schematic diagram of a cluster of resonant scatterers located at arbitrary positions. Each scatterer is numbered by a Greek letter. The inset shows the parameters corresponding to a single scatterer. A 2-beam resonator is illustrated as a typical example. (b) Scheme of a slab with a thickness of three layers but infinite along the $y$ direction. The scatterers are arranged in a square lattice. Each scatterer is numbered by a couple of letters defining the row and column. On both figures, a plane wave impinges the structures with an angle of incidence $\theta_{0}$.

field inside the inner plate is

$$
W^{I}(r, \theta)=\sum_{q=-\infty}^{\infty} F_{q}^{J I}\left(k_{p} r, \theta\right) C_{q},
$$

where the superscript $I$ is used to distinguishing the out-of-plane displacements in the region $I, W^{I}$, from their counterparts defined in region $I I$, and $C_{q}=\left[C_{q}^{J} C_{q}^{I}\right]^{t}$ contains the expansion coefficients for flexural waves inside the region $I$. In numerical calculations, the series have to be truncated and the summations run from $-N_{q}$ to $N_{q}$, where the value $N_{q}$ giving adequate accuracy is determined through the convergence study. ${ }^{26}$

For the beams in one resonator, the transverse (out-of-plane) displacement of the $n$-th beam $V^{n}(x)$ is controlled by the following equation ${ }^{29}$

$$
\frac{\partial^{4} V^{n}}{\partial x^{4}}-k_{b}^{4} V^{n}=0
$$

where the beam is defined for $x \in\left[R_{1}, R_{2}\right]$ with an inclination angle of $\theta^{n}(n=1$ to $N)$. The wave number in the beam is $k_{b}=\left(m \omega^{2} / E \mathrm{I}\right)^{1 / 4}$, with $\mathrm{I}=b h^{3} / 12$ being the second moment 
of area and $m=\rho b h$ being the mass per unit length. The solution of Eq. (6) is

$$
V^{n}(x)=D_{1}^{n} e^{i k_{b} x}+D_{2}^{n} e^{-i k_{b} x}+D_{3}^{n} e^{k_{b} x}+D_{4}^{n} e^{-k_{b} x}
$$

where coefficients $D_{i}^{n}(i=1$ to 4$)$ define the contributions of the different waves inside the beams.

\section{B. Multiple scattering theory for arbitrarily located scatterers}

Consider a cluster of scatterers arbitrarily located on the plate, as shown in Fig. 1(a), $\boldsymbol{R}_{\alpha}$ $\left(\alpha=1\right.$ to $\left.N_{s c}\right)$ defines the position of the center of the $\alpha$ scatterer in the global coordinates system $(R, \Theta)$. If an external wave $W^{0}$ impinges on the cluster, the total scattered field is obtained by adding all the scattered fields by each scatterer

$$
W^{s c a t}(R, \Theta)=\sum_{\alpha=1}^{N_{s c}} \sum_{q=-\infty}^{\infty} F_{q}^{H K}\left(k_{p} r_{\alpha}, \theta_{\alpha}\right)\left(B_{\alpha}\right)_{q},
$$

where the column matrix $\left(B_{\alpha}\right)_{q}$ contains the expansion coefficients for waves scattered by the $\alpha$ scatterer. The polar coordinates $\left(r_{\alpha}, \theta_{\alpha}\right)$ are measured in a local system centered at $\boldsymbol{R}_{\alpha}$. In order to avoid any confusion, the uppercase and lowercase letters are adopted to distinguish the polar coordinates used in the global and local systems. As shown in Fig. 1(a), the field point $P$ can be expressed in the global and local systems through $\boldsymbol{R}=\boldsymbol{R}_{\alpha}+\boldsymbol{r}_{\alpha}$.

Multiple scattering theory is a self-consistent theory in which the incoming wave of an arbitrary scatterer $\alpha$ is made of a combination of the external waves and scattered waves caused by all the remaining scatterers

$$
\begin{aligned}
W_{\alpha}^{i n c}\left(r_{\alpha}, \theta_{\alpha}\right) & =\sum_{s=-\infty}^{\infty} F_{s}^{J I}\left(k_{p} r_{\alpha}, \theta_{\alpha}\right)\left(A_{\alpha}\right)_{s} \\
& =\sum_{s=-\infty}^{\infty} F_{s}^{J I}\left(k_{p} R, \Theta\right) A_{s}^{0}+\sum_{\beta=1}^{N_{s c}} \sum_{s=-\infty}^{\infty}\left(1-\delta_{\alpha \beta}\right) F_{s}^{H K}\left(k_{p} r_{\beta}, \theta_{\beta}\right)\left(B_{\beta}\right)_{s},
\end{aligned}
$$

where $\left(A_{\alpha}\right)_{s}$ includes the total incoming coefficients for $\alpha$ scatterer and $A_{s}^{0}$ contains the coefficients for external waves expanded in the global system. For a plane wave, $W^{0}=e^{i \boldsymbol{k}_{p} \cdot \boldsymbol{R}}$, the expansion coefficients are $A_{s}^{0}=\left[\begin{array}{ll}i^{s} e^{-i s \theta_{0}} & 0\end{array}\right]^{t}$ with $\theta_{0}$ being the propagation angle. After introducing the Graf's addition theorem, ${ }^{28,30}$ the total incoming coefficients $\left(A_{\alpha}\right)_{s}$ can be expressed in the $\alpha$ frame as

$$
\left(A_{\alpha}\right)_{s}=A_{s}^{0} e^{i \boldsymbol{k}_{p} \cdot \boldsymbol{R}_{\alpha}}+\sum_{\beta=1}^{N_{s c}} \sum_{r=-\infty}^{\infty}\left(1-\delta_{\alpha \beta}\right) \boldsymbol{G}_{s r}\left(k_{p} R_{\alpha \beta}, \Theta_{\alpha \beta}\right)\left(B_{\beta}\right)_{r},
$$


where the matrix $\boldsymbol{G}_{s r}$ is related with the frame change from $\beta$ scatterer to $\alpha$ scatterer

$$
\boldsymbol{G}_{s r}\left(k_{p} R_{\alpha \beta}, \Theta_{\alpha \beta}\right)=\left[\begin{array}{cc}
H_{s-r}\left(k_{p} R_{\alpha \beta}\right) & 0 \\
0 & (-1)^{r} K_{r-s}\left(k_{p} R_{\alpha \beta}\right)
\end{array}\right] e^{i(r-s) \Theta_{\alpha \beta}} .
$$

The coefficients of the incoming and scattered waves for the $\alpha$ scatterer are linked through the transfer matrix

$$
\left(B_{\alpha}\right)_{q}=\sum_{s=-\infty}^{\infty}\left(\boldsymbol{T}_{\alpha}\right)_{q s}\left(A_{\alpha}\right)_{s}
$$

where $\boldsymbol{T}_{\alpha}$ is the transfer matrix of the $\alpha$ resonator and its specific form can be found in Ref. 26. Substituting Eq. (10) into Eq. (12), we have

$$
\left(B_{\alpha}\right)_{q}=\sum_{s=-\infty}^{\infty}\left(\boldsymbol{T}_{\alpha}\right)_{q s} A_{s}^{0} e^{i \boldsymbol{k}_{p} \cdot \boldsymbol{R}_{\alpha}}+\sum_{\beta=1}^{N_{s c}} \sum_{r, s=-\infty}^{\infty}\left(1-\delta_{\alpha \beta}\right)\left(\boldsymbol{T}_{\alpha}\right)_{q s} \boldsymbol{G}_{s r}\left(k_{p} R_{\alpha \beta}, \Theta_{\alpha \beta}\right)\left(B_{\beta}\right)_{r} .
$$

After a few operations, the linear system of equations can be expressed as

$$
\sum_{\beta=1}^{N_{s}} \sum_{r=-\infty}^{\infty}\left(\boldsymbol{M}_{\alpha \beta}\right)_{q r}\left(B_{\beta}\right)_{r}=\left(N_{\alpha}\right)_{q}
$$

with notations

$$
\begin{gathered}
\left(\boldsymbol{M}_{\alpha \beta}\right)_{q r}=\delta_{\alpha \beta} \delta_{q r} \boldsymbol{I}-\sum_{s=-\infty}^{\infty}\left(1-\delta_{\alpha \beta}\right)\left(\boldsymbol{T}_{\alpha}\right)_{q s} \boldsymbol{G}_{s r}\left(k_{p} R_{\alpha \beta}, \Theta_{\alpha \beta}\right) \\
\left(N_{\alpha}\right)_{q}=\sum_{s=-\infty}^{\infty}\left(\boldsymbol{T}_{\alpha}\right)_{q s} A_{s}^{0} e^{i \boldsymbol{k}_{p} \cdot \boldsymbol{R}_{\alpha}}
\end{gathered}
$$

where $\delta$ is the Kronecker symbol and $\boldsymbol{I}$ is the unit matrix with dimension $2 \times 2$.

The coefficients for all scatterers can be solved from Eq. (14), then the total field is easily available by adding external waves to the total scattered waves, namely

$$
W(R, \Theta)=e^{i \boldsymbol{k}_{p} \cdot \boldsymbol{R}}+\sum_{\alpha=1}^{N_{s c}} \sum_{q=-\infty}^{\infty} F_{q}^{H K}\left(k_{p} r_{\alpha}, \theta_{\alpha}\right)\left(B_{\alpha}\right)_{q} .
$$

Alternatively, the total field can be expressed in a local frame by considering the scattering process for one particular scatterer, take $\alpha$ scatterer as an example, we have

$$
W\left(r_{\alpha}, \theta_{\alpha}\right)=\sum_{q=-\infty}^{\infty}\left[F_{q}^{J I}\left(k_{p} r_{\alpha}, \theta_{\alpha}\right)\left(A_{\alpha}\right)_{q}+F_{q}^{H K}\left(k_{p} r_{\alpha}, \theta_{\alpha}\right)\left(B_{\alpha}\right)_{q}\right],
$$

The second form expressed in the above equation will be employed to solve the coefficients for waves in the inner plates and beams. 
For $N$-beam resonators, the fields in the inner plates are determined by the following procedures: ${ }^{26}$

$$
W_{\alpha}^{I}\left(r_{\alpha}, \theta_{\alpha}\right)=\sum_{q=-\infty}^{\infty} F_{q}^{J I}\left(k_{p} r_{\alpha}, \theta_{\alpha}\right)\left(C_{\alpha}\right)_{q}
$$

where

$$
\left(C_{\alpha}\right)_{q}=\sum_{s=-\infty}^{\infty}\left(\hat{\boldsymbol{T}}_{\alpha}\right)_{q s}\left[\begin{array}{c}
W \\
\frac{\partial W}{\partial r_{\alpha}}
\end{array}\right]_{s} .
$$

The matrix $\hat{\boldsymbol{T}}_{\alpha}$ is closely related with the geometric parameters of the $\alpha$ resonator and the reader is recommended to the original paper for more information. The column matrix appearing in the right hand side of Eq. (20) is rewritten, according to Eq. (18), as

$$
\left[\begin{array}{c}
W \\
\frac{\partial W}{\partial r_{\alpha}}
\end{array}\right]_{s}=\boldsymbol{M}_{s s}^{J I}\left(k_{p}, R_{2}\right)\left(A_{\alpha}\right)_{s}+\boldsymbol{M}_{s s}^{H K}\left(k_{p}, R_{2}\right)\left(B_{\alpha}\right)_{s}
$$

where the additional matrices appearing are defined as

$$
M_{s s}^{\Upsilon \Phi}\left(k_{i}, r\right)=\left[\begin{array}{cc}
\Upsilon_{s}\left(k_{i} r\right) & \Phi_{s}\left(k_{i} r\right) \\
k_{i} \Upsilon_{s}^{\prime}\left(k_{i} r\right) & k_{i} \Phi_{s}^{\prime}\left(k_{i} r\right)
\end{array}\right] .
$$

Finally, the multiple scattering problem is fully solved by giving the coefficients determining the flexural waves in the $n$-th beam of the $\alpha$ scatterer, it is

$$
\begin{aligned}
{\left[\begin{array}{c}
\left(D_{\alpha}^{n}\right)_{1} \\
\left(D_{\alpha}^{n}\right)_{2} \\
\left(D_{\alpha}^{n}\right)_{3} \\
\left(D_{\alpha}^{n}\right)_{4}
\end{array}\right]=\boldsymbol{H}^{-1} \sum_{q=-\infty}^{\infty}\left[\begin{array}{cc}
\mathbf{0} & \mathbf{0} \\
\boldsymbol{M}_{q q}^{J I}\left(k_{p}, R_{2}\right) & \boldsymbol{M}_{q q}^{H K}\left(k_{p}, R_{2}\right)
\end{array}\right]\left[\begin{array}{c}
\left(A_{\alpha}^{J}\right)_{q} \\
\left(A_{\alpha}^{I}\right)_{q} \\
\left(B_{\alpha}^{H}\right)_{q} \\
\left(B_{\alpha}^{K}\right)_{q}
\end{array}\right] e^{i q \theta_{\alpha}^{n}} } \\
+\boldsymbol{H}^{-1} \sum_{q=-\infty}^{\infty}\left[\begin{array}{cc}
\boldsymbol{M}_{q q}^{J I}\left(k_{p}, R_{1}\right) & \mathbf{0} \\
\mathbf{0} & \mathbf{0}
\end{array}\right]\left[\begin{array}{c}
\left(C_{\alpha}^{J}\right)_{q} \\
\left(C_{\alpha}^{I}\right)_{q} \\
0 \\
0
\end{array}\right] e^{i q \theta_{\alpha}^{n}},
\end{aligned}
$$

where $\boldsymbol{H}$ is an auxiliary matrix employed in expressing the beam stiffness matrix and the explicit expression can be found in the original paper. ${ }^{26}$

\section{Scattering by a slab with a finite number of layers}

Here an infinite slab is considered to analyze the reflectance and transmittance when a plane wave is impinging on it. The slab consists of $L$ layers in the $x$-direction and is infinite 
in the $y$-direction. The distances between the two nearest scatterers in each row and column are denoted as $d_{x}$ and $d_{y}$, respectively. For simplicity, it is assumed that all the resonators are identical and the position of a given scatterer is defined by a pair of indices with the numbers of each row and column denoted by a Greek letter and a Latin letter, respectively. The column index runs from 1 to $L$ and the row index runs from $-\infty$ to $\infty$. As shown in Fig. 1(b), the quantities involved in the previous derivation are renamed with the new notations defined here. For example, $\boldsymbol{R}_{\alpha}^{l}$ is the position vector measured in the global system for the scatterer located in $l$ column and $\alpha$ row, particularly, $\boldsymbol{R}_{0}^{l}$ defines the center of this column. The field point $P$, which is defined as $(R, \Theta)$ in the global system, is measured as $\left(r_{\alpha}^{l}, \theta_{\alpha}^{l}\right)$ in a local frame centered at $\boldsymbol{R}_{\alpha}^{l}$.

The procedures explained in Sec. IIB can be easily applied to the present case by updating the labels of each scatterer, subsequently, Eq. (13) is rewritten as

$$
\begin{aligned}
\left(B_{\alpha}^{l}\right)_{q}=\sum_{s=-\infty}^{\infty} \boldsymbol{T}_{q s} A_{s}^{0} e^{i \boldsymbol{k}_{p} \cdot \boldsymbol{R}_{0}^{l}} e^{i \alpha k_{p} d_{y} \sin \theta_{0}} & \\
& +\sum_{m=1}^{L} \sum_{\beta=-\infty}^{\infty} \sum_{r, s=-\infty}^{\infty}\left(1-\delta_{l m} \delta_{\alpha \beta}\right) \boldsymbol{T}_{q s} \boldsymbol{G}_{s r}\left(k_{p} R_{\alpha \beta}^{l m}, \Theta_{\alpha \beta}^{l m}\right)\left(B_{\beta}^{m}\right)_{r},
\end{aligned}
$$

where the phase factor $e^{i \alpha k_{p} d_{y} \sin \theta_{0}}$ is introduced by substituting the geometric relation $\boldsymbol{R}_{\alpha}^{l}=$ $\boldsymbol{R}_{0}^{l}+\boldsymbol{R}_{0 \alpha}^{l l}$. Notice that this equation still takes into account the infinite number of cylinders and the problem can be simplified by considering the symmetry of the geometry model here studied. Multiplying Eq. (24) by $e^{-i \alpha k_{p} d_{y} \sin \theta_{0}}$, then we have

$$
\begin{aligned}
& {\left[\left(B_{\alpha}^{l}\right)_{q} e^{-i \alpha k_{p} d_{y} \sin \theta_{0}}\right]=\sum_{s=-\infty}^{\infty} \boldsymbol{T}_{q s} A_{s}^{0} e^{i \boldsymbol{k}_{p} \cdot \boldsymbol{R}_{0}^{l}}} \\
& \quad+\sum_{m=1}^{L} \sum_{\beta=-\infty}^{\infty} \sum_{r, s=-\infty}^{\infty}\left(1-\delta_{l m} \delta_{\alpha \beta}\right) \boldsymbol{T}_{q s} \boldsymbol{G}_{s r}\left(k_{p} R_{\alpha \beta}^{l m}, \Theta_{\alpha \beta}^{l m}\right) e^{i(\beta-\alpha) k_{p} d_{y} \sin \theta_{0}}\left[\left(B_{\beta}^{m}\right)_{r} e^{-i \beta k_{p} d_{y} \sin \theta_{0}}\right]
\end{aligned}
$$

It is clear that the first term in the right hand side is independent on $\alpha$, and we make it a priori for the other terms in a similar way. Then a new set of coefficients is defined as

$$
\left(B_{0}^{m}\right)_{r}=\left(B_{\beta}^{m}\right)_{r} e^{-i \beta k_{p} d_{y} \sin \theta_{0}}
$$

where the newly defined matrix contains the scattering coefficients of the central scatterer 
in each column. Finally, the counterparts of Eqs. (14), (15) and (16) are

$$
\sum_{m=1}^{L} \sum_{r=-\infty}^{\infty}\left(\boldsymbol{M}^{l m}\right)_{q r}\left(B_{0}^{m}\right)_{r}=\left(N_{0}^{l}\right)_{q}
$$

where

$$
\begin{gathered}
\left(\boldsymbol{M}^{l m}\right)_{q r}=\delta_{l m} \delta_{q r} \boldsymbol{I}-\sum_{\beta=-\infty}^{\infty} \sum_{s=-\infty}^{\infty}\left(1-\delta_{l m} \delta_{0 \beta}\right) \boldsymbol{T}_{q s} \boldsymbol{G}_{s r}\left(k_{p} R_{0 \beta}^{l m}, \Theta_{0 \beta}^{l m}\right) e^{i \beta k_{p} d_{y} \sin \theta_{0}} \\
\left(N_{0}^{l}\right)_{q}=\sum_{s=-\infty}^{\infty} \boldsymbol{T}_{q s} A_{s}^{0} e^{i \boldsymbol{k}_{p} \cdot \boldsymbol{R}_{0}^{l}}
\end{gathered}
$$

For solving efficiently the infinite sum in $\beta$, we express the matrix as a combination of complete sums and incomplete sums. The reader is addressed to Appendices A and B for more details regarding the calculations of lattice sums and matrix $\boldsymbol{M}$.

Through the system defined in Eqs. (27), (28) and (29), we get the scattering coefficients for the central scatterers $(\beta=0)$ of each column and, subsequently, the coefficients for the remaining scatterers $(\beta \neq 0)$ are obtained through the relationship defined in Eq. (26). Finally, the total displacement field expressed in Eq. (17) is rewritten as

$$
W(R, \Theta)=e^{i \boldsymbol{k}_{p} \cdot \boldsymbol{R}}+\sum_{q=-\infty}^{\infty} \sum_{l=1}^{L}\left[\sum_{\alpha=-\infty}^{\infty} e^{i \alpha k_{p} d_{y} \sin \theta_{0}} F_{q}^{H K}\left(k_{p} r_{\alpha}^{l}, \theta_{\alpha}^{l}\right)\right]\left(B_{0}^{l}\right)_{q},
$$

where the position vector $\boldsymbol{r}_{\alpha}^{l}\left(r_{\alpha}^{l}, \theta_{\alpha}^{l}\right)$ is defined in the local frame as

$$
\boldsymbol{r}_{\alpha}^{l}=\boldsymbol{R}-\boldsymbol{R}_{0}^{l}-\boldsymbol{R}_{0 \alpha}^{l l}
$$

Introducing the lattice sums defined in Appendix A, Eq. (30) can be rewritten as series summation with physical meaning. Then, the total field is

$$
W(R, \Theta)=e^{i \boldsymbol{k}_{p} \cdot \boldsymbol{R}}+\sum_{\sigma=-\infty}^{\infty}\left(\zeta_{\sigma}^{H} e^{i \boldsymbol{\kappa}_{\sigma} \cdot \boldsymbol{R}}+\zeta_{\sigma}^{K} e^{-\gamma_{\sigma} \cdot \boldsymbol{R}}\right)
$$

where

$$
\begin{aligned}
& \zeta_{\sigma}^{H}=\sum_{q=-\infty}^{\infty} \sum_{l=1}^{L} \frac{2 i^{-q}}{k_{p} d_{y}} \frac{e^{i q \tau_{1}(\sigma)}}{\left|\cos \tau_{1}(\sigma)\right|} e^{-i \boldsymbol{\kappa}_{\sigma} \cdot \boldsymbol{R}_{0}^{l}}\left(B_{0}^{l}\right)_{q}^{H}, \\
& \zeta_{\sigma}^{K}=\sum_{q=-\infty}^{\infty} \sum_{l=1}^{L} \frac{\pi}{k_{p} d_{y}} \frac{e^{i q \tau_{2}(\sigma)}}{\left|\cos \tau_{2}(\sigma)\right|} e^{\gamma_{\sigma} \cdot \boldsymbol{R}_{0}^{l}}\left(B_{0}^{l}\right)_{q}^{K} .
\end{aligned}
$$


The meaning of each quantity in the previous equations can be found in Appendix A. Once the total field is determined, we can obtain the displacements of the inner plates and beams following the formulas listed in Eqs. (19)-(23).

To better understand the physical meaning of the previous results, the energy flux flowing through the vertical segment $\left[y, y+d_{y}\right]$ is analytically calculated. The time averaged flux $\left\langle F_{ \pm}\right\rangle$is defined as ${ }^{31}$

$$
\left\langle F_{ \pm}\right\rangle= \pm \frac{\omega D}{2} \int_{y}^{y+d_{y}} \operatorname{Im}\left[W \frac{\partial}{\partial x}\left(\triangle W^{*}\right)-\left(\triangle W^{*}\right) \frac{\partial W}{\partial x}\right] d y
$$

where ' $\triangle$ ' and ' $*$ ' represent the Laplace operator and complex conjugate, respectively. The subscript ' ' is used to distinguish the flux flowing along and opposite to the positive $x$-direction. The energy flux of the incoming plane wave, $W^{0}=e^{i \boldsymbol{k}_{p} \cdot \boldsymbol{R}}$, is calculated as $\left\langle F_{0}\right\rangle=\omega d_{y} D k_{p}^{3} \cos \theta_{0}$, and the energy flux of the total field under study is derived as (see Appendix C)

$$
\left\langle F_{ \pm}\right\rangle= \pm \omega d_{y} D k_{p}^{3}\left\{\cos \theta_{0}+(1 \pm 1) \operatorname{Re}\left[\zeta_{0}^{H}\right] \cos \theta_{0} \pm \sum_{\cos \tau_{1}(\sigma) \in \mathbb{R}}\left|\zeta_{\sigma}^{H}\right|^{2}\left|\cos \tau_{1}(\sigma)\right|\right\} .
$$

Dividing the flux of the total field by the incoming one, the reflectance and the transmittance are calculated as

$$
\begin{gathered}
R=1+\frac{\left\langle F_{-}\right\rangle}{\left\langle F_{0}\right\rangle}=\sum_{\cos \tau_{1}(\sigma) \in \mathbb{R}}\left|\zeta_{\sigma}^{H}\right|^{2} \frac{\left|\cos \tau_{1}(\sigma)\right|}{\cos \theta_{0}} \\
T=\frac{\left\langle F_{+}\right\rangle}{\left\langle F_{0}\right\rangle}=1+2 R e\left[\zeta_{0}^{H}\right]+\sum_{\cos \tau_{1}(\sigma) \in \mathbb{R}}\left|\zeta_{\sigma}^{H}\right|^{2} \frac{\left|\cos \tau_{1}(\sigma)\right|}{\cos \theta_{0}} .
\end{gathered}
$$

For an infinite slab without dissipation, the sum of reflectance and transmittance equals to one as a result of energy conservation. This condition has been employed to check the accuracy of the numerical results.

From the results in Eqs. (32) and (36), we can conclude that the total field is composed of the incident plane wave and a series of scattered waves containing propagating waves (with real $\boldsymbol{\kappa}_{\sigma}$ ) and evanescent waves (with complex $\boldsymbol{\kappa}_{\sigma}$ ). Only the propagating waves transport energy. The evanescent waves are responsible only for the surface effects. If real $\boldsymbol{\kappa}_{\sigma}$ is achieved with more than one values of $\sigma$, the incoming energy is distributed among these different order of diffracted waves. Particularly, it is worth mentioning the critical mode in which the $x$-component of diffracted wave vector $\boldsymbol{\kappa}_{\sigma}$ is vanishing. In this case, the order of scattered wave is under a transition from propagating to evanescent and, consequently, the Rayleigh anomalies occurring. ${ }^{32,33}$ 


\section{Band structure calculation}

Let us consider any of the 2D Bravais lattices containing a scatterer per unit cell. The lattice constant is $d$ and we assume that a scatterer is placed at the origin of the global system of coordinates. Each scatterer is labeled by a pair of indices as explained in Sec. IIC, but now the column index runs from $-\infty$ to $\infty$. According to Bloch's theorem, the total field can be expressed as a plane wave modulated by the periodicity of the lattice

$$
W\left(\boldsymbol{R}+\boldsymbol{R}_{\beta}^{m}\right)=e^{i \boldsymbol{K} \cdot \boldsymbol{R}_{\beta}^{m}} W(\boldsymbol{R}),
$$

where $\boldsymbol{K}$ is the Bloch vector. The solutions of interest are the eigenmodes of the periodic system, in other words, no incident field is considered, as a consequence, we obtain the following relationship linking the scattering coefficients of resonators located at the origin and $\boldsymbol{R}_{\beta}^{m}$

$$
\left(B_{\beta}^{m}\right)_{q}=e^{i \boldsymbol{K} \cdot \boldsymbol{R}_{\beta}^{m}} B_{q}
$$

With this relation and reminding that the external wave is null, we have after updating the indices in Eq. (14)

$$
\sum_{r=-\infty}^{\infty} M_{q r} B_{r}=0
$$

where the $\boldsymbol{M}$ matrix is

$$
\boldsymbol{M}_{q r}=\delta_{q r} \boldsymbol{I}-\sum_{s=-\infty}^{\infty} \sum_{m, \beta=-\infty}^{\infty}\left(1-\delta_{0 \beta} \delta_{0 m}\right) \boldsymbol{T}_{q s} \boldsymbol{G}_{s r}\left(k_{p} R_{\beta}^{m}, \Theta_{\beta}^{m}\right) e^{i \boldsymbol{K} \cdot \boldsymbol{R}_{\beta}^{m}}
$$

Notice that the double summations in $m$ and $\beta$ converge very slowly when computing the $M$ matrix, hence the lattice sums are employed to improve the computing efficiency. ${ }^{34}$ In order to obtain non-trivial solutions, the determinant of $\boldsymbol{M}$ must be zero. This defines the secular equation of the periodic system and the band structure can be obtained by running the Bloch vector $\boldsymbol{K}$ along the edges of the irreducible Brillouin zone. For each Bloch vector, a series of trial frequencies are employed in computing $\boldsymbol{M}$ and the ones that make the matrix tend to be singular are considered as the approximate solutions. This algorithm needs many trial frequencies to get solutions enough accurate. Therefore, it is less efficient in computing the whole band structures when compared with the well-developed commercial finite element packages. Nevertheless, the utility of the method here developed cannot be neglected in certain situations. For example, the method shows its advantages in computing 
the refractive index at low frequencies (see Fig. 8), where only one value on the first band is needed in each calculation.

\section{RESULTS AND DISCUSSION}

The algorithms introduced above can be used as a powerful tool to predict the propagation properties of flexural waves in thin plates with $N$-beam resonators. As a typical example of application, in what follows we comprehensively study the properties of 2-beam resonators arranged in a square lattice with lattice constant $d$. In fact, this type of structure has been experimentally analyzed showing directional propagation. ${ }^{25}$ Therefore, for comparison purposes, the relevant physical and geometric parameters employed for the numerical calculation are taken directly from Ref. 25: $E=69 \mathrm{GPa}, \nu=0.33, \rho=2.7 \times 10^{3} \mathrm{~kg} / \mathrm{m}^{3}$, $R_{1}=5 \mathrm{~mm}, R_{2}=10 \mathrm{~mm}, b=2 \mathrm{~mm}, h=1 \mathrm{~mm}$ and $d=25 \mathrm{~mm}$. The truncation order is set as $N_{q}=18$, which is chosen according to the results of the convergence study performed for a single resonator. ${ }^{26}$

\section{A. Band structure for a square lattice of 2-beam resonators}

The procedure explained in Sec. IID has been applied to obtain the band structure of a square lattice of 2-beam resonators with parameters as described above. Figure 2 shows

the calculated band structure (solid circles). In brief, the Bloch vector $\boldsymbol{K}$ is scanned along the edges of the irreducible Brillouin zone, which is shown in the inset. The OA and OC directions represent the situations where the beams are aligned along the $x$ - and $y$-axis, respectively. For the sake of comparison, the hollow circles represent the band structure obtained with a 3D full elastic model; i.e., using the commercial software COMSOL which is based on the finite element method. On the other hand, the numerical results reported in Ref. 25, which were obtained by using Mindlin plate elements, will be also discussed in what follows.

The full elastic equations are employed in solving the 3D model so that both the inplane and out-of-plane motions are taken into consideration. It is observed from Fig. 2 that additional bands appear in the 3D calculation when compared with our theoretical results. Except for the flat band labeled as $\mathbf{b}$, in the low frequency region, the additional 


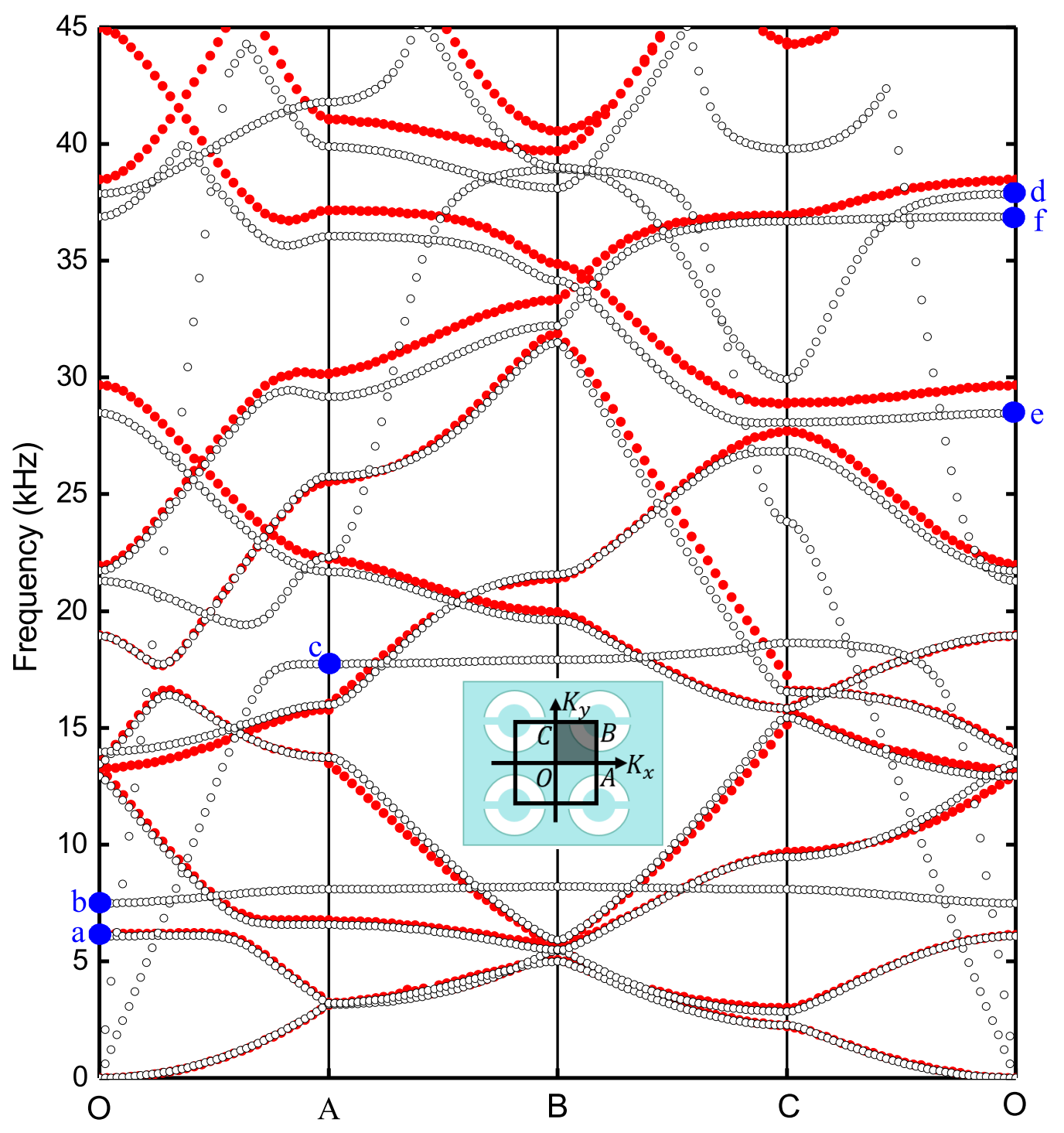

FIG. 2. (color on line) Band structure of a square lattice of 2-beam resonators. The dispersion relation obtained with the 2D model here introduced (red solid circles) is compared with that obtained using a 3D finite element method (hollow circles). The inset shows a scheme of the lattice in real space together with the definitions of the high symmetry directions in $\mathbf{K}$-space, where the irreducible Brillouin zone is depicted in gray. Bands of interest are marked out at selected points a-f.

bands correspond to in-plane motions. Then, regarding the flexural modes, we can conclude that our 2D modeling is in good agreement with the 3D full elastic model for frequencies below $25 \mathrm{kHz}$, or in reduced units $k_{p} R_{2} \leq \pi$. For frequencies above $25 \mathrm{kHz}$, however, large discrepancies start to appear. It is reasonable to attribute the discrepancies to convergence 
issues due to truncation in the series summations. In other words, more terms should be employed when computing the series. This calculation has been omitted due to the computational time.

The flat bands appearing in the band structure indicate the occurrence of some kinds of local resonances. Figure 3 displays the eigenmodes at selected points (see labels a-f in Fig. 2) within these flat bands. Figures 3(a)-3(f) are obtained with the finite element commercial software and they show a 3D representation of eigenmodes at points a-f, respectively. It is observed in Figs. 3(a)-3(d) that some resonances are associated with movements inside the hole while the background plate remains almost motionless. The inner plate experiences strong deformations, representing flexural, torsional, translational and rotational displacements, respectively.

For the flexural mode represented in Fig. 3(a) we observe that the inner plate vibrates as a whole and the beams behave like extension springs providing the restoring force for the mass in a spring-mass resonator. A simple spring-mass resonator model (see Appendix D) gives that the frequency of this fundamental mode is $5891 \mathrm{~Hz}$. This frequency matches quite well with the eigenfrequency at point a in Fig. 2, which is $6078 \mathrm{~Hz}$. In fact, this resonance represents the fundamental mode for $N$-beam resonators and has been studied in a previous work by analyzing the scattering cross-section. ${ }^{26}$ We found that the fundamental resonance of the 2-beam resonator with same geometry appears at $6175 \mathrm{~Hz}$, which is in good agreement with the aforementioned frequencies.

For the torsional mode shown in Fig. 3(b), the beams experience torsional deformation, behaving like torsion springs. Moreover, the inner plate rotates as a whole in the out-ofplane direction, the axis of rotation being the beams. The beam theory fails in this case and the flat band associated with this kind of resonance is missing in the band structure obtained by our 2D model. However, Mindlin plate theory doesn't have this limitation, so the flat band was perfectly reproduced in Andreassen and coworkers' work. $^{25}$

The eigenmodes represented in Figs. 3(c) and 3(d) correspond to in-plane motions, which are out of the scope of the present work. However, it is worth mentioning that they have interesting properties for designing elastic metamaterials; for example, these two resonances have been employed to design negative refraction lens for longitudinal waves in solids. ${ }^{4}$ Quite differently, the eigenmodes displayed in Figs. 3(e) and 3(f) show antiresonance features because the inner plate is motionless. Meanwhile, it is observed that the edges of the unit 


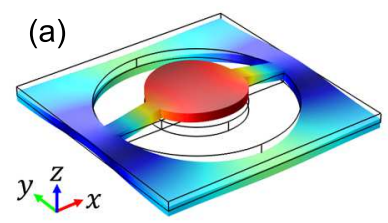

(b)

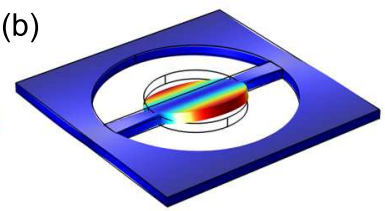

(c)

(d)

(e)

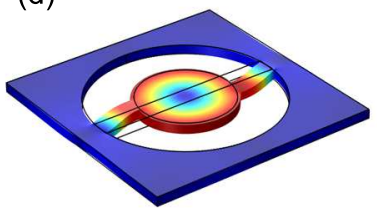

(e)

(g)

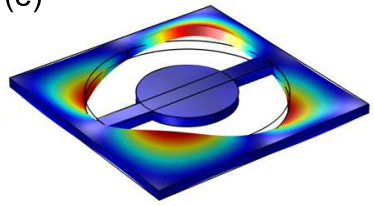

(h)
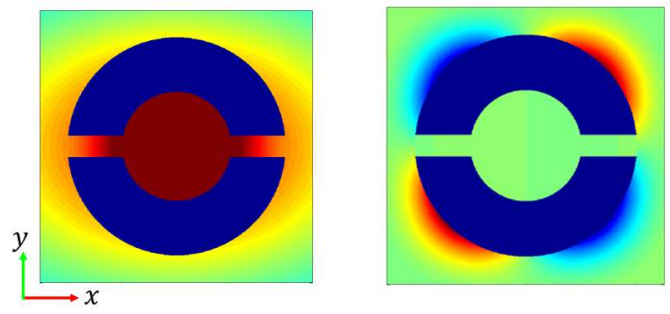

(i)

(f)
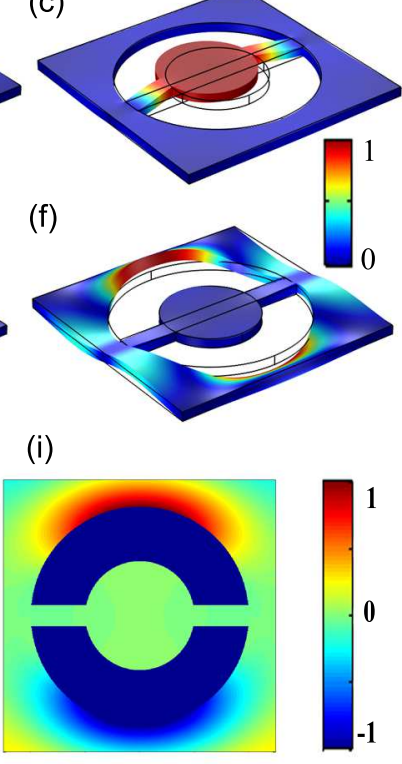

FIG. 3. (color on line) Eigenmodes at selected points within the flat bands (see labels a-f in Fig. 2). The eigenmodes in (a)-(f) are 3D representations and correspond to the eigenmodes at the points a-f in Fig. 2. They are obtained with a commercial software using a 3D finite elements method. The 2D displacement maps shown in (g)-(i) represent the eigenmodes obtained with our multiple scattering approach and they reproduce fairly well the modes at points $\mathbf{a}$, e and $\mathbf{f}$, respectively.

cell are almost stationary. This characteristic is similar to the nodes of standing waves propagating in a string and indicates that no energy can pass the nodal lines, which is the underlying mechanism for the flat dispersion relation. The last row of graphs, corresponding to Figs. 3(g)-3(i), represent 2D displacement maps of the flexural eigenmodes at points a, e and f, respectively. They are obtained with the 2D multiple scattering model developed above. From their comparison with the corresponding 3D representations we can conclude that our 2D model is enough accurate to predict the propagation behavior of flexural waves in perforated plates.

From Fig. 2 we observe that the structure under study does not contain complete bandgaps. Nevertheless, partial bandgaps or pseudogaps are observed along specific directions as a result of Bragg scattering and local resonances. Now, attention is paid to the band structure along the OA direction, which is shown in the left panel of Fig. 4. For comparison purposes, the band structure for a square lattice of empty holes (hollow circles) 
is also plotted. It is noticeable that, in the low frequency region, the dispersion relation corresponding to the lattice of 2-beam resonators shows an additional band in comparison with the lattice of empty holes. This band is strictly associated to the lowest resonant mode embedded in the scattering unit. This fundamental mode creates a narrow resonant bandgap due to its interaction with flexural waves propagating in the plate. The bandgap defined at points $\mathbf{a}-\mathbf{b}$ corresponds to that situation. On the other hand, the bandgap at high frequencies, with edges at the points e-f, represents a regular bandgap associated to Bragg scattering. Finally, the pseudogap defined by the anticrossing bands at points $\mathbf{c}-\mathbf{d}$ is not of interest here and will not be further discussed. It is originated by the interaction of modes with similar symmetry but belonging to different bands.

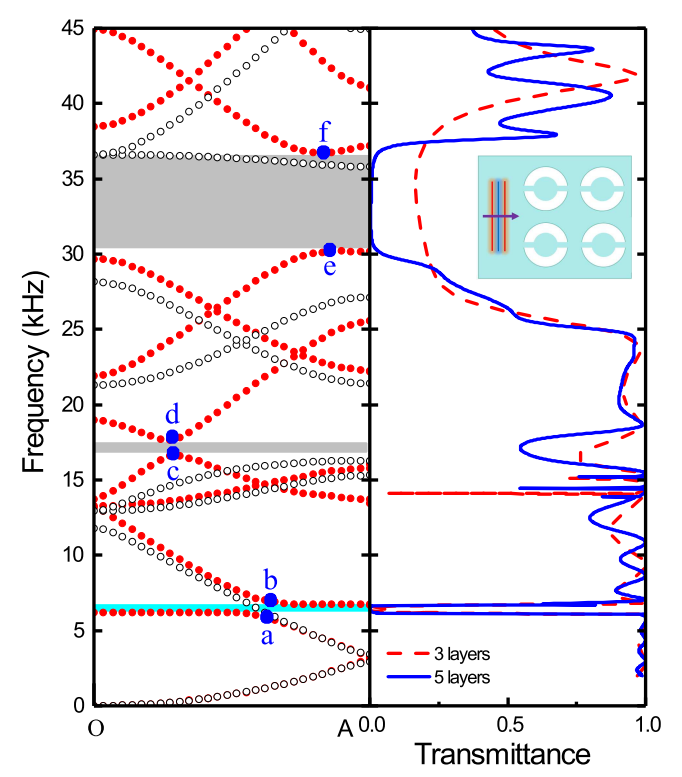

FIG. 4. (color on line) Left panel: Dispersion relation of flexural waves propagating in a thin plate containing a square lattice of 2-beam resonators (solid circles). The hollow circles represent the dispersion corresponding to a square lattice of empty holes with same radii $R_{2}$. For frequencies below $5 \mathrm{kHz}$ both dispersion relations are almost identical. Right panel: Transmittance spectra obtained for slabs made of three (dashed line) and five (solid line) layers. The inset shows that resonators are oriented in the same direction as the propagating wave, the $x$-direction. The slabs are infinite along the $y$-direction.

In order to demonstrate the existence of the pseudogaps observed in the band structure, we have studied the transmission through finite crystal structures orientated along the OA 

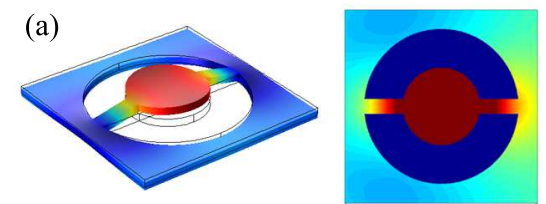

(c)

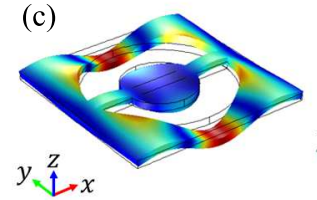

(b)

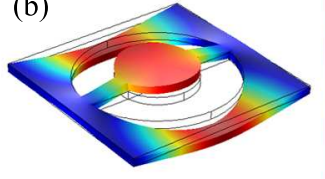

(d)

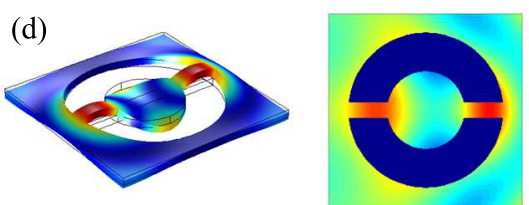

FIG. 5. (color on line) Eigenmodes calculated at the edges of two bandgaps appearing in the band structure shown in Fig. 4. The 3D plots are obtained using a 3D finite element method (left panels) while the 2D maps are obtained using the 2D multiple scattering method here reported (right panels). (a)-(b) Eigenmodes at points a and b in Fig. 4. (c)-(d) Eigenmodes at points e and $\mathbf{f}$ in Fig. 4.

direction. The right panel in Fig. 4 shows the transmittance spectra calculated using the numerical algorithm described in Sec. II C. The spectra corresponding to slabs made of three and five layers are depicted as dashed and solid lines, respectively. As expected, it is shown that bandgaps correspond to minimums in the transmittance spectra. It is also observed that bandgaps are better defined with increasing number of layers of the crystal slab. For example, five layers are needed in order to forbid completely the propagation of flexural waves in the region between $30.2 \mathrm{kHz}$ and $36.7 \mathrm{kHz}$, corresponding to a Bragg bandgap. However, for the low frequency bandgap, corresponding to a resonant bandgap, three layers are enough to stop the propagation between $5.9 \mathrm{kHz}$ and $7.0 \mathrm{kHz}$.

Figure 5 displays the eigenmodes associated with the edges of the two partial gaps of interest here; see points $\mathbf{a}-\mathbf{b}$ and $\mathbf{e - f}$, respectively, in Fig. 4. The 3D and 2D representations correspond to results obtained from a 3D finite element model and our 2D analytical model, respectively. For the low frequency gap [see Figs. 5(a) and 5(b)], the fundamental mode of the 2-beam resonator interacts with flexural waves propagating in the plate, producing the bandgap opening. Notice that this behavior is specific for this type of scattering unit, not appearing for the case of empty holes scatterers [see Fig. 4]. Conversely, if a local resonance doesn't interact with propagating waves in the plate, the energy remains in the resonators and no gap can be opened. This behavior is observed for the flat band labeled as $\mathbf{b}$ in Fig. 2; it corresponds to a torsional resonance [see Fig. 3(b)] not interacting with 
flexural waves. The modes associated with the high frequency bandgap [see Figs. 5(c) and $5(\mathrm{~d})$ ] show different features as the dynamic deformation is no longer localized in the inner structure, but occurs over the whole unit cell, which is the typical feature of Bragg-type bandgaps.

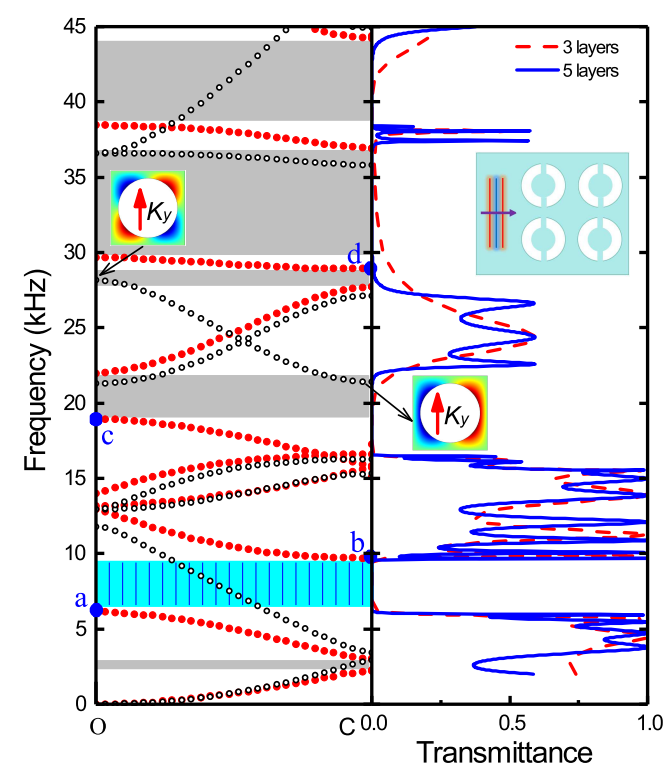

FIG. 6. (color on line) Left panel: Dispersion relation of the flexural waves propagating along the $\mathrm{OC}$ direction in a thin plate containing a square lattice of 2-beam resonators (solid circles). The hollow circles represent the dispersion corresponding to a square lattice of empty holes with same radii $R_{2}$. Right panel: Transmittance spectra obtained for slabs made of three (dashed line) and five (solid line) layers. The inset shows that the resonators are orientated perpendicularly to the propagating wave. The slabs are infinite along the $y$-direction.

For its interest, let us discuss the band structure along the OC direction, which is shown in Fig. 6. Compared with the previous case, additional bandgaps appear along this direction and their existence is proved again by the transmittance dips observed in the spectra. A bandgap appears at low frequencies but now is much broader than its counterpart along the OA direction [see Fig. 4]. The frequencies at the lower and upper edges are $6.1 \mathrm{kHz}$ and $9.6 \mathrm{kHz}$, respectively. This bandgap enhancement is attributed to the stronger interaction between the local resonance with the propagating flexural waves; now the beams are excited in phase because they are arranged in parallel with the wavefronts of the incident wave. Regarding high frequency bandgaps, it is observed that minimums in the transmittance 

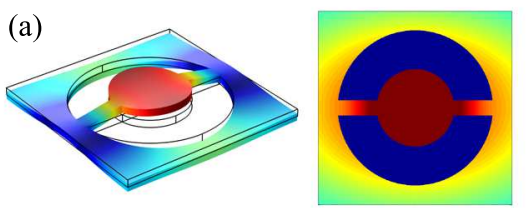

(c)

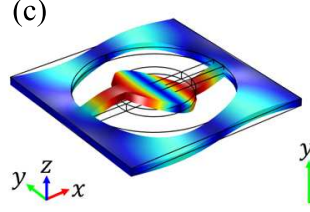

(b)

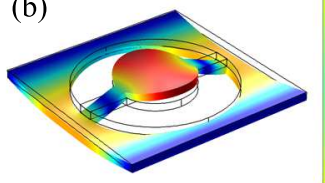

(d)

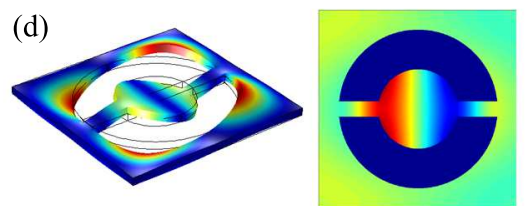

FIG. 7. (color on line) (a)-(b) Eigenmodes at the edges of the resonance-type bandgap [see points $\mathbf{a}$ and $\mathbf{b}$ in Fig. 6]. (c)-(d) Eigenmodes at selected points within the deaf bands [see points $\mathbf{c}$ and d in Fig. 6]. The 3D representations obtained with a 3D finite element method (left panels) are compared with the 2D maps obtained with our 2D multiple scattering model (right panels).

are broader than that predicted by the band structure calculation. This effect can be explained in terms of the existence in the dispersion relation of deaf bands, ${ }^{35}$ labeled as $\mathbf{c}$ and $\mathbf{d}$ in Fig. 6, located near the bandgaps. Therefore, since the modes in these bands are antisymmetric with respect to the incident wave, they cannot be excited and create additional non-propagating frequency regions in the transmittance spectra.

The eigenmodes calculated at selected points in the band structure [see labels a to $\mathbf{d}$ in Fig. 6] are depicted in Fig. 7. On the one hand, Figs. 7(a) and 7(b) depict the eigenmodes at the edges of the resonance-type bandgap, showing the excitation of the fundamental mode of scattering unit. On the other hand, the eigenmodes in Figs. 7(c) and 7(d) belong to deaf bands. Notice that they are antisymmetric with respect to the direction of the impinging wave. ${ }^{35,36}$ The deaf bands cannot be excited by the external wave and, consequently, they produce additional minimums in the transmission spectra.

\section{B. Gradient index lens made of 2-beam resonators arrays}

The first bands in Fig. 4 and Fig. 6 follow a parabolic dispersion relation; i.e., $\omega=\beta k^{2}$. However, the $\beta$ coefficient depends on the relative orientation of the 2-beam resonator with respect to the propagation direction in the plate. In other words, the dispersion relation is anisotropic and a homogenization procedure can be applied to design gradient index materials by tuning the orientation of 2-beam resonators. 


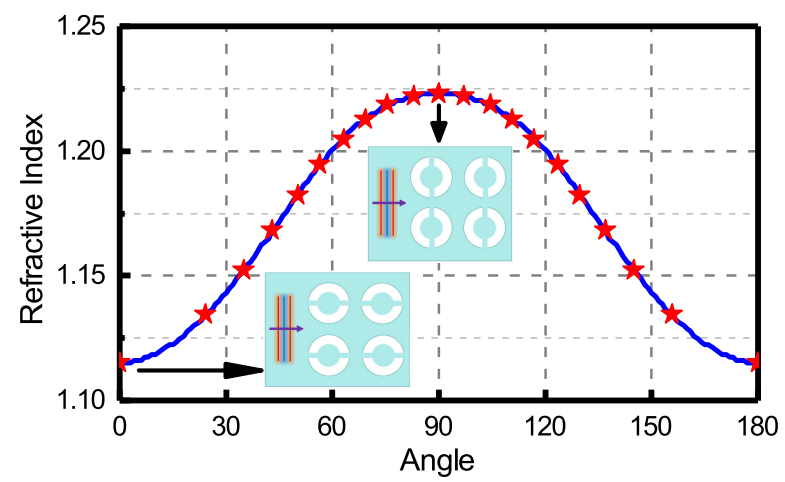

FIG. 8. (color on line) Effective refractive index as a function of the angle between the beams and the incident direction. The insets show the two limiting cases: $0^{\circ}$ and $90^{\circ}$. The stars symbols define the discrete values employed in designing the gradient index lens.

Based on the Snell's law, the refractive index for flexural waves with frequencies in the first band can be defined as

$$
n=\sqrt{\frac{\beta_{i}}{\beta_{t}}},
$$

where $\beta_{i}$ and $\beta_{t}$ are the coefficients for materials in the incident side and transmitted side, respectively. Within the first band, these coefficients are easily obtained and then the refractive index. In brief, for each angle of tilting (i.e., the angle between the beams and the incoming waves), only one point in the first band is needed to get its corresponding $\beta$. Thus, for the case of 2-beam resonators, the continuous line in Fig. 8 represents the dependence of $n$ with the tilted angle. The refractive indices $n_{x}$ and $n_{y}$ are defined when the wave is incoming from the $x$ - and $y$-directions, respectively. Therefore, they are identical since the angle in Fig. 8 represents the tilted angle between the beams and the incoming waves. A symmetric profile is obtained owing to the geometric symmetry. We can conclude that the minimum and maximum values of $n$ are obtained when the beams are parallel and perpendicularly, respectively, to the incident direction [see the insets of Fig. 8]. Between the two limiting cases ( 0 and 90 degrees), the refractive index $n$ increases with the angle of tilting.

In what follows, the homogenization described above is applied to design a gradient index lens. In previous works, ${ }^{7-9}$ the hyperbolic secant profile has been chosen to obtain focusing with less aberration

$$
n(y)=n(0) \operatorname{sech}(\alpha y),
$$




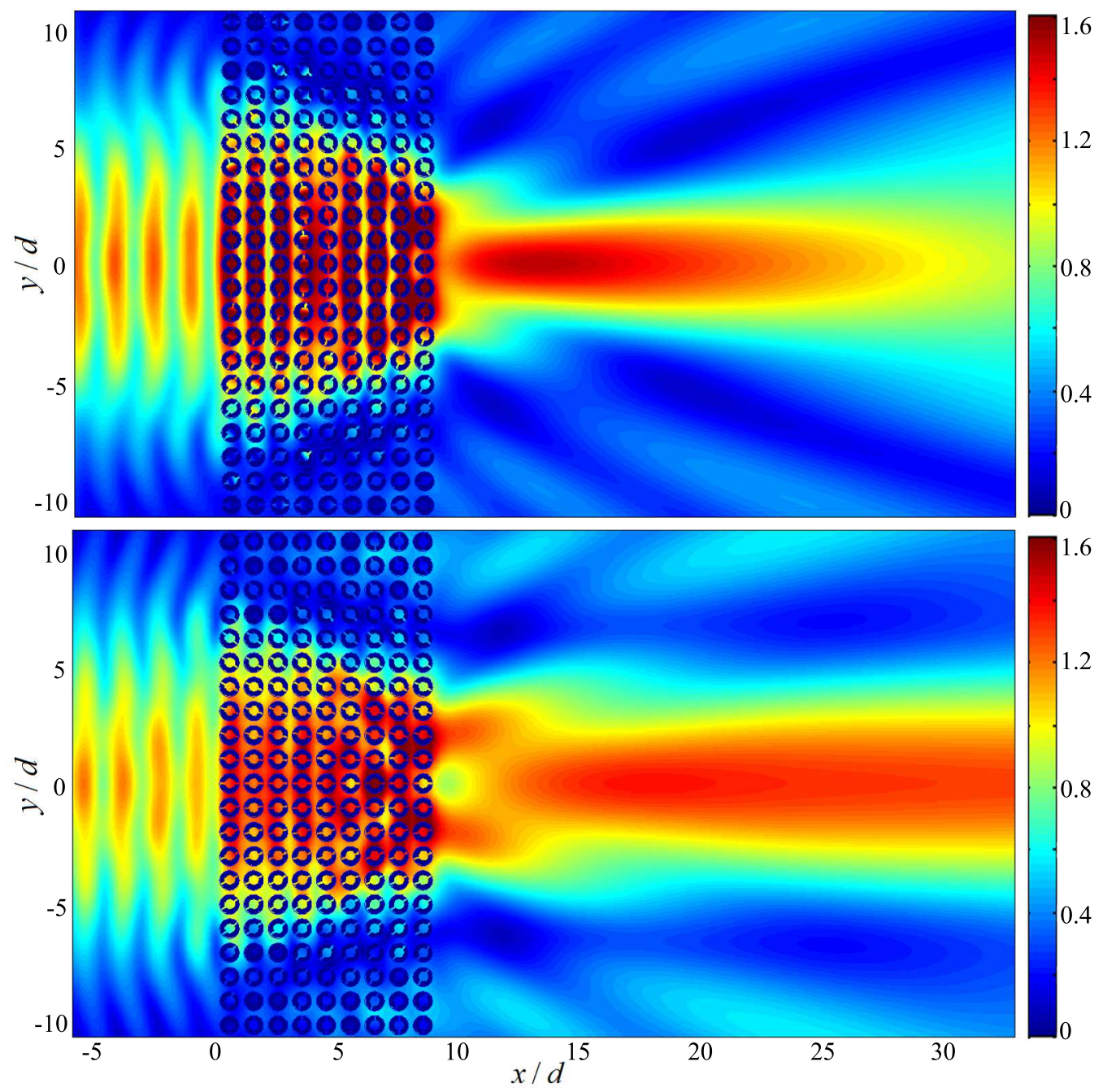

FIG. 9. (color on line) 2D maps of the (out-of-plane) displacement amplitude for the $x$-dominated lens (top panel) and $y$-dominated lens (bottom panel). The frequency of the incident Gaussian beam is $1.6 \mathrm{kHz}$.

where the gradient index is

$$
\alpha=\frac{1}{\ell} \cosh ^{-1}\left[\frac{n(0)}{n( \pm \ell)}\right]
$$

where $\ell$ is the half-height of the lens, $n(0)$ is the refractive index on the $x$-axis $(y=0)$ and $n( \pm \ell)$ is that at the ends of the lens $(y= \pm \ell)$.

In principle, we would like to get the profile described in Eq. (44) by changing the orientation of beams. The profile should be isotropic theoretically, but this limitation has to be discarded here because the anisotropy is the underlying mechanism employed to achieve the gradient index. Then, the profile is obtained by changing the $x$ and $y$ components of 
refractive index, $n_{x}(y)$ and $n_{y}(y)$, individually. For the sake of simplicity, the two kinds of lenses are called as $x$-dominated lens and $y$-dominated lens, respectively. Both are designed with profiles as closer as possible to that of Eq. (44) with $n(0)=1.223$ and $n( \pm \ell)=1.115$. The designed devices consist of 9 column layers and each layer contains 21 resonators. All the resonators are arranged in a square lattice with lattice constant $d=25 \mathrm{~mm}$. The tilted angles in each column are marked out with stars symbols in Fig. 8. Then, the values from the left to the right in Fig. 8 correspond to the resonators from the top to the bottom in Fig. 9. Let us remark that, for $n_{x}\left(n_{y}\right)$, the horizontal axis in Fig. 8 represents the angle between the beams and the $x$-axis ( $y$-axis).

The performance of the proposed devices has been studied using the multiple scattering algorithm reported in Sec. IIB. A Gaussian beam with amplitude unity along its central axis is employed in the calculation. Figure 9 reports the $2 \mathrm{D}$ maps corresponding to the displacement amplitude of the $x$-dominated lens (top panel) and $y$-dominated lens (bottom panel) for the common frequency of $1.6 \mathrm{kHz}$. In both figures, the coordinates are normalized to the lattice constant. It is noticed that the focusing behavior is observed in both cases and the amplitude amplification at the focal points are 1.5 ( $x$-dominated lens) and 1.3 ( $y$ dominated lens). Larger amplitude amplification is obtained from the $x$-dominated lens and the focal region of the $y$-dominated lens is much broader.

The anisotropy presents two main aspects: on one hand, it enables to tune the refractive index by changing the orientation of beams and thus achieving focusing by gradient index lenses; on the other hand, it results in an unavoidable aberration, the position of the focal point being frequency dependent. To better illustrate this effect, Fig. 10 shows the amplitude distribution for several frequencies for the the $x$-dominated lens (a) and $y$-dominated lens (b), respectively. The displacement amplitudes are normalized to the maximum amplitude of the Gaussian beam. The shadowed regions bound the positions of the focal points for the frequencies studied. It is observed that the calculated profiles match well with the experimental profiles obtained with acoustic convergent lens. ${ }^{37}$ All in all, the $x$-dominated lens performs better than the $y$-dominated lens. First, the aberration of the $y$-dominated lens is far greater than that of $x$-dominated lens. Besides, for the $x$-dominated lens, the positions of the focal points shift within two times the lattice constant for the frequencies studied. The shifting is almost eight times for the $y$-dominated lens. Based on this analysis, it is concluded that the $x$-dominated lens is a good alternative to be used in energy harvesting 

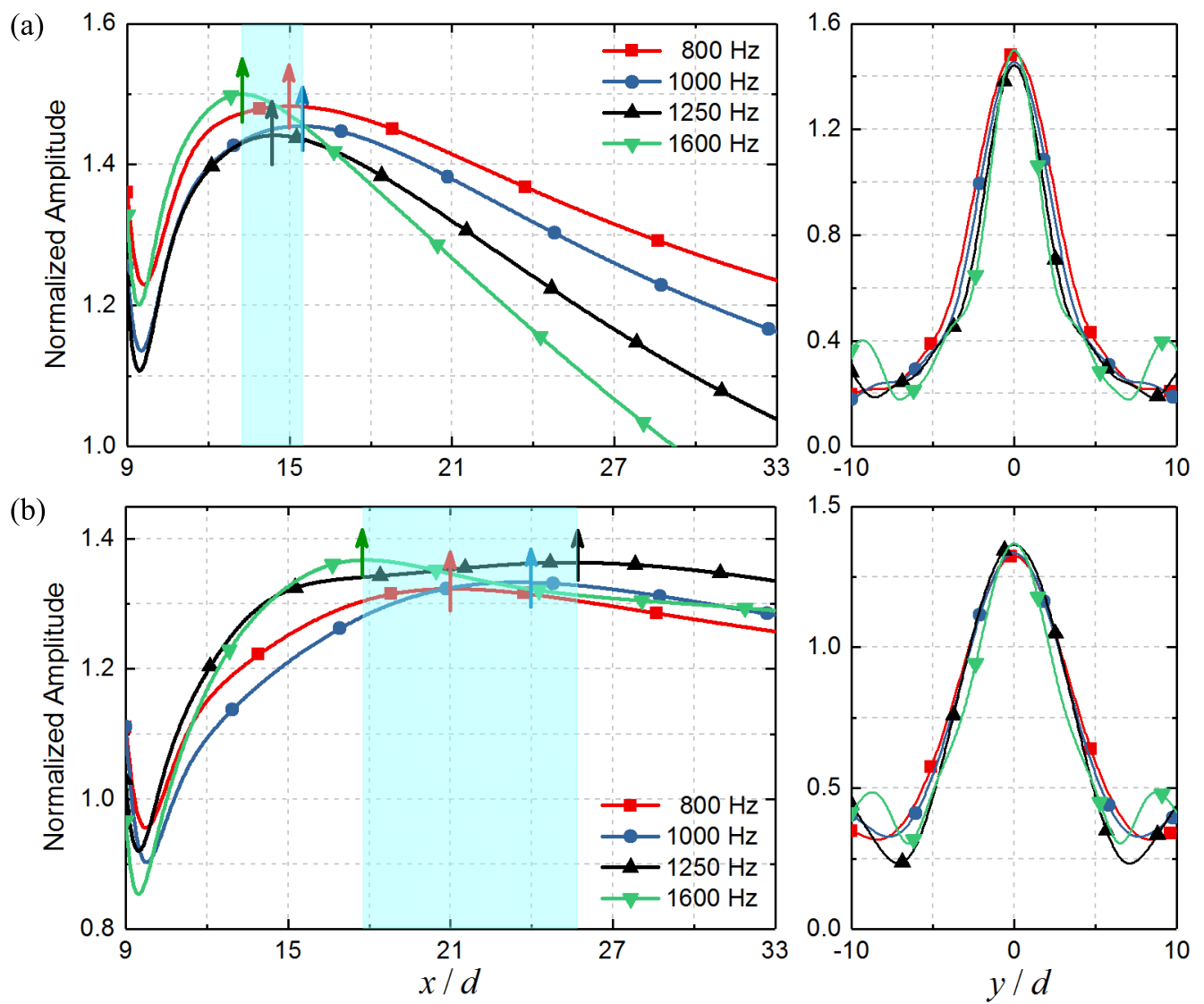

FIG. 10. (color on line) Left panels: The normalized amplitude distribution along the $x$-axis for the $x$-dominated lens (a) and $y$-dominated lens (b), respectively. The arrows indicate the positions of the focal points. The shadowed regions define the band where the focal points are found for the frequencies studied. Right panels: The normalized amplitude distribution in the $y$-direction at the focal points.

for flexural waves even though the scheme here proposed should be improved. ${ }^{14,15}$

\section{SUMMARY}

In summary, within the framework on the multiple scattering theory, this work has theoretically investigated the behavior of flexural wave propagating in thin perforated plates containing $N$-beam resonators. We have developed a numerical algorithm allowing the computation of the flexural band structures in lattices containing a resonator per unit cell. Also, a semi-analytical method allowing the calculation of the transmittance spectrum through several layers of resonators has been reported. Both algorithms have been successfully em- 
ployed to comprehensively study, as an example, a square lattice of 2-beam resonators. The resulting band structures and eigenmodes are in agreement with 3D full elastic model as long as the truncation order $N_{q}$ of the series summation is large enough to meet the required accuracy. Of course, our modeling is subjected to the limitations of the Kirchhoff-Love and the Euler-Bernoulli theories and, therefore, torsional modes and in-plane propagation are features not covered by our algorithms.

We have also demonstrated that the fundamental mode of the 2-beam resonator can be modeled by simple spring-mass oscillator. It has been shown that a low frequency bandgap appears as a consequence of the the interaction between this fundamental mode and the propagating flexural waves. In addition, as an application of our modeling we have designed gradient index lenses for flexural waves in which the local refractive index is obtained by changing the orientation of the resonator with respect to the direction of the impinging wave. In summary, we can conclude that the theoretical tools here developed can be used to tackle a variety of problems within the field of elastic metamaterials based on the $N$-beam resonators. The demonstration of negative refraction based on them is one goal of to be tackled in the near future.

\section{ACKNOWLEDGEMENTS}

This work was supported by the Ministerio de Economía y Competitividad of the Spanish government and the European Union Fondo Europeo de Desarrollo Regional (FEDER) through Project No. TEC2014-53088-C3-1-R, and the National Science Foundation of China under Grant No. 11432004. Penglin Gao acknowledges a scholarship with No. 201606120070 provided by China Scholarship Council.

\section{Appendix A: Lattice sums}

Several complex quantities containing infinite series summations are involved in the derivation. The complete sums and incomplete sums appear when studying the transmission and reflection of flexural waves scattered by an infinite slab. They are defined, respectively, 
as

$$
\begin{aligned}
& S_{q}^{c H}\left(k_{p} d_{y}, \sin \theta_{0}, \boldsymbol{R}, \boldsymbol{R}_{0}^{l}\right)=\sum_{\alpha=-\infty}^{\infty} e^{i \alpha k_{p} d_{y} \sin \theta_{0}} H_{q}\left(k_{p} r_{\alpha}^{l}\right) e^{i q \theta_{\alpha}^{l}}, \\
& S_{q}^{c K}\left(k_{p} d_{y}, \sin \theta_{0}, \boldsymbol{R}, \boldsymbol{R}_{0}^{l}\right)=\sum_{\alpha=-\infty}^{\infty} e^{i \alpha k_{p} d_{y} \sin \theta_{0}} K_{q}\left(k_{p} r_{\alpha}^{l}\right) e^{i q \theta_{\alpha}^{l}},
\end{aligned}
$$

and

$$
\begin{aligned}
& S_{q}^{i H}\left(k_{p} d_{y}, \sin \theta_{0}\right)=\sum_{\alpha=1}^{\infty} e^{i \alpha k_{p} d_{y} \sin \theta_{0}} H_{q}\left(\alpha k_{p} d_{y}\right), \\
& S_{q}^{i K}\left(k_{p} d_{y}, \sin \theta_{0}\right)=\sum_{\alpha=1}^{\infty} e^{i \alpha k_{p} d_{y} \sin \theta_{0}} K_{q}\left(\alpha k_{p} d_{y}\right) .
\end{aligned}
$$

Notice that the complete sums $S_{q}^{c H}$ and $S_{q}^{c K}$ are position dependent and the geometric quantities involved are related through the relationship defined in Eq. (31). Moreover, the lattice sums are introduced when computing band structures for an infinite lattice, they are defined for Hankel function and modified Bessel function, respectively, as

$$
S_{q}^{H}\left(k_{p}, \boldsymbol{K}\right)=\sum_{m=-\infty}^{\infty} \sum_{\beta=-\infty}^{\infty}\left(1-\delta_{0 m} \delta_{0 \beta}\right) H_{q}\left(k_{p} R_{\beta}^{m}\right) e^{i q \Theta_{\beta}^{m}} e^{i \boldsymbol{K} \cdot \boldsymbol{R}_{\beta}^{m}}
$$

and

$$
S_{q}^{K}\left(k_{p}, \boldsymbol{K}\right)=\sum_{m=-\infty}^{\infty} \sum_{\beta=-\infty}^{\infty}\left(1-\delta_{0 m} \delta_{0 \beta}\right) K_{q}\left(k_{p} R_{\beta}^{m}\right) e^{i q \Theta_{\beta}^{m}} e^{i \boldsymbol{K} \cdot \boldsymbol{R}_{\beta}^{m}} .
$$

Because the modified Bessel function is exponentially decaying with the increasing of argument $\alpha k_{p} d_{y}$, the summation defined in Eq. (A.4) converges rapidly and therefore can be evaluated by direct summation. Except this, other summation series defined in the previous equations converge very slowly so that some other expressions have to be found for practical calculation.

For Eqs. (A.1) and (A.3), an alternative form has been provided by Torrent ${ }^{38}$ when dealing with the homogenization problem of a cluster of cylinders for acoustic waves. The result corresponding to Eq. (A.1) is

$$
S_{q}^{c H}=\frac{2 i^{-q}}{k_{p} d_{y}} \sum_{\sigma=-\infty}^{\infty} \frac{e^{i q \tau_{1}(\sigma)}}{\left|\cos \tau_{1}(\sigma)\right|} e^{i \boldsymbol{\kappa}_{\sigma} \cdot\left(\boldsymbol{R}-\boldsymbol{R}_{0}^{l}\right)},
$$

where the quantities involved are defined as

$$
\boldsymbol{\kappa}_{\sigma}=k_{p}\left[\cos \tau_{1}(\sigma), \sin \tau_{1}(\sigma)\right]
$$




$$
\begin{gathered}
e^{i q \tau_{1}(\sigma)}=\left[\cos \tau_{1}(\sigma)+i \sin \tau_{1}(\sigma)\right]^{q} \\
\sin \tau_{1}(\sigma)=\sin \theta_{0}-\sigma \frac{2 \pi}{k_{p} d_{y}} \\
\cos \tau_{1}(\sigma)= \pm \sqrt{1-\sin ^{2} \tau_{1}(\sigma)} .
\end{gathered}
$$

The alternative expression of Eq. (A.3) is

$$
S_{q}^{i H}=\frac{i^{-q} e^{-\frac{\pi}{4} i}}{\pi} \int_{-\infty}^{\infty} \frac{e^{i k_{p} d_{y} \sin \theta_{0}} e^{i k_{p} d_{y} \sqrt{1+i \tau^{2}}}}{1-e^{i k_{p} d_{y} \sin \theta_{0}} e^{i k_{p} d_{y} \sqrt{1+i \tau^{2}}}} \frac{\left(\sqrt{1+i \tau^{2}}+i \tau e^{-\frac{\pi}{4} i}\right)^{q}}{\sqrt{1+i \tau^{2}}} d \tau .
$$

It is suggested that the integral should be managed cautiously in the numerical calculation because the integrand involved is an intensively oscillating function. The simplified version of lattice sums defined in Eqs. (A.5) and (A.6) has been reported as well when studying Bloch-Floquet bending waves in perforated thin plates. ${ }^{34}$ They are clearly explained and the reader is recommended to the original paper for more information in details.

Concerning the last quantity defined in Eq. (A.2), let us begin with the integral definition of modified Bessel function $K^{39}$

$$
K_{q}(x)=\frac{1}{2} \lim _{\epsilon \rightarrow \infty} \int_{-\epsilon}^{\epsilon} e^{-x \cosh t} e^{q t} d t
$$

where the integration path is from $-\infty$ to $\infty$. We change the variable with relation $t=i \tau$, and the integral is rewritten as

$$
K_{q}(x)=-\frac{i}{2} \lim _{\epsilon \rightarrow \infty} \int_{-i \epsilon}^{i \epsilon} e^{-x \cos \tau} e^{i q \tau} d \tau
$$

To obtain the general functions needed, we introduce the variable changes $\tau=\tau_{2}-\theta_{\alpha}^{l}$ and $x=k_{p} r_{\alpha}^{l}$, consequently, we obtain the following form

$$
K_{q}\left(k_{p} r_{\alpha}^{l}\right) e^{i q \theta_{\alpha}^{l}}=-\frac{i}{2} \lim _{\epsilon \rightarrow \infty} \int_{-i \epsilon+\theta_{\alpha}^{l}}^{i \epsilon+\theta_{\alpha}^{l}} e^{-\gamma \cdot r_{\alpha}^{l}} e^{i q \tau_{2}} d \tau_{2}
$$

where $\gamma=k_{p}\left(\cos \tau_{2}, \sin \tau_{2}\right)$. According to Cauchy's theorem, the integration limits can be replaced by taken a different path of integration, the path $C$ is from $z_{1}=-i \infty$ to $z_{2}=i \infty$ when $\cos \theta_{\alpha}^{l} \geq 0$ and from $z_{1}=\pi-i \infty$ to $z_{2}=\pi+i \infty$ when $\cos \theta_{\alpha}^{l} \leq 0$. Multiplying Eq. (A.15) by $e^{i \alpha k_{p} d_{y} \sin \theta_{0}}$ and adding the terms for all $\alpha$, finally, we obtain the desired form of Eq. (A.2) as

$$
S_{q}^{c K}=\sum_{\alpha=-\infty}^{\infty}-\frac{i}{2} \int_{C} e^{-\gamma \cdot r_{\alpha}^{l}} e^{i \alpha k_{p} d_{y} \sin \theta_{0}} e^{i q \tau_{2}} d \tau_{2}
$$


Taking the geometric relations into consideration

$$
\boldsymbol{\gamma} \cdot \boldsymbol{r}_{\alpha}^{l}=\gamma \cdot\left(\boldsymbol{R}-\boldsymbol{R}_{0}^{l}\right)-\alpha k_{p} d_{y} \sin \tau_{2}
$$

then we have the following equation

$$
S_{q}^{c K}=-\frac{i}{2} \int_{C} e^{-\boldsymbol{\gamma} \cdot\left(\boldsymbol{R}-\boldsymbol{R}_{0}^{l}\right)} e^{i q \tau_{2}} \sum_{\alpha=-\infty}^{\infty} e^{i \alpha k_{p} d_{y}\left(\sin \theta_{0}-i \sin \tau_{2}\right)} d \tau_{2} .
$$

The series summation in the integrand can be expressed as a Dirac comb

$$
\sum_{\alpha=-\infty}^{\infty} e^{i \alpha k_{p} d_{y}\left(\sin \theta_{0}-i \sin \tau_{2}\right)}=\frac{2 \pi}{k_{p} d_{y}} \sum_{\sigma=-\infty}^{\infty} \delta\left(\sin \theta_{0}-i \sin \tau_{2}-\frac{2 \pi \sigma}{k_{p} d_{y}}\right) .
$$

In order to use the properties of Dirac function, it is necessary to convert the integration path from the complex plane to the real axis. The variables are changed through the relation $t_{2}=i \sin \tau_{2}$, correspondingly, the integration limits are changed from $\infty(-\infty)$ to $-\infty(\infty)$ when $\cos \theta_{\alpha}^{l} \geq 0\left(\cos \theta_{\alpha}^{l} \leq 0\right)$. Then the new form of Eq. (A.18) is

$$
S_{q}^{c K}=\frac{\pi}{k_{p} d_{y}} \sum_{\sigma=-\infty}^{\infty} \int_{-\infty}^{\infty} e^{-\boldsymbol{\gamma} \cdot\left(\boldsymbol{R}-\boldsymbol{R}_{0}^{l}\right)} e^{i q \tau_{2}\left(t_{2}\right)} \delta\left(\sin \theta_{0}-t_{2}-\frac{2 \pi \sigma}{k_{p} d_{y}}\right) \frac{d t_{2}}{\sqrt{1+t_{2}^{2}}} .
$$

Evaluating the Dirac function at $t_{2}=\sin \theta_{0}-\frac{2 \pi \sigma}{k_{p} d_{y}}$, we eventually obtain the new form of the complete sum as

$$
S_{q}^{c K}=\frac{\pi}{k_{p} d_{y}} \sum_{\sigma=-\infty}^{\infty} \frac{e^{i q \tau_{2}(\sigma)}}{\left|\cos \tau_{2}(\sigma)\right|} e^{-\gamma_{\sigma} \cdot\left(\boldsymbol{R}-\boldsymbol{R}_{0}^{l}\right)},
$$

where the quantities involved are defined as

$$
\begin{gathered}
\gamma_{\sigma}=k_{p}\left[\cos \tau_{2}(\sigma), \sin \tau_{2}(\sigma)\right], \\
e^{i q \tau_{2}(\sigma)}=\left[\cos \tau_{2}(\sigma)+i \sin \tau_{2}(\sigma)\right]^{q}, \\
\sin \tau_{2}(\sigma)=-i\left(\sin \theta_{0}-\sigma \frac{2 \pi}{k_{p} d_{y}}\right), \\
\cos \tau_{2}(\sigma)= \pm \sqrt{1-\sin ^{2} \tau_{2}(\sigma)}
\end{gathered}
$$

The sign of $\cos \tau_{2}(\sigma)$ is positive (negative) when $x-x_{0}^{l}$ is positive (negative), where $x$ comes from $\boldsymbol{R}=x \hat{\boldsymbol{x}}+y \hat{\boldsymbol{y}}$ and $x_{0}^{l}$ comes from $\boldsymbol{R}_{0}^{l}=x_{0}^{l} \hat{\boldsymbol{x}}+y_{0}^{l} \hat{\boldsymbol{y}}$. 


\section{Appendix B: Simplification for $M$ matrix}

The matrix provided in Eq. (28) is of complex form containing an infinite sum in $\beta$, we are going to simplify it by dividing it into two different cases. Let us begin by rewriting the equation as

$$
\left(\boldsymbol{M}^{l m}\right)_{q r}=\delta_{l m} \delta_{q r} \boldsymbol{I}-\sum_{s=-\infty}^{\infty} \boldsymbol{T}_{q s} \mathcal{G}_{s r}
$$

where the $2 \times 2$ matrix $\mathcal{G}_{s r}$ is

$$
\mathcal{G}_{s r}=\sum_{\beta=-\infty}^{\infty}\left(1-\delta_{l m} \delta_{0 \beta}\right) \boldsymbol{G}_{s r}\left(k_{p} R_{0 \beta}^{l m}, \Theta_{0 \beta}^{l m}\right) e^{i \beta k_{p} d_{y} \sin \theta_{0}} .
$$

In the first case, $l=m$, the pervious equation can be expressed in an explicit form after using the definition of $\boldsymbol{G}_{s r}$ from Eq. (11)

$$
\mathcal{G}_{s r}=\sum_{\beta=-\infty}^{\infty}\left[\begin{array}{cc}
H_{s-r}\left(k_{p} R_{0 \beta}^{l l}\right) & 0 \\
0 & (-1)^{r} K_{r-s}\left(k_{p} R_{0 \beta}^{l l}\right)
\end{array}\right]\left(1-\delta_{0 \beta}\right) e^{i(r-s) \Theta_{0 \beta}^{l l} e^{i \beta k_{p} d_{y} \sin \theta_{0}}}
$$

Note that $R_{0 \beta}^{l l}=|\beta| d_{y}$ and $\Theta_{0 \beta}^{l l}=\pi / 2\left(\Theta_{0 \beta}^{l l}=-\pi / 2\right)$ when $\beta>0(\beta<0)$, after some operations and then using the definitions of incomplete sums, finally, we obtain

$$
\left(\boldsymbol{M}^{l m}\right)_{q r}=\delta_{q r} \boldsymbol{I}-\sum_{s=-\infty}^{\infty} \boldsymbol{T}_{q s}\left[\begin{array}{cc}
\mathcal{G}_{s r}^{i H} & 0 \\
0 & \mathcal{G}_{s r}^{i K}
\end{array}\right]
$$

where

$$
\begin{aligned}
& \mathcal{G}_{s r}^{i H}=(-i)^{r-s} S_{r-s}^{i H}\left(k_{p} d_{y}, \sin \theta_{0}\right)+i^{r-s} S_{r-s}^{i H}\left(k_{p} d_{y},-\sin \theta_{0}\right), \\
& \mathcal{G}_{s r}^{i K}=(-1)^{s}\left[(-i)^{r-s} S_{r-s}^{i K}\left(k_{p} d_{y}, \sin \theta_{0}\right)+i^{r-s} S_{r-s}^{i K}\left(k_{p} d_{y},-\sin \theta_{0}\right)\right] .
\end{aligned}
$$

The other case is $l \neq m$, then Eq. (B.2) can be simplified by using the complete sums defined in Appendix A. Before this, it is necessary to find the correspondences between the quantities involved. From Fig. 1(b), we can easily obtain the following relationships

$$
\begin{gathered}
\boldsymbol{R}-\boldsymbol{r}_{\alpha}^{l}=\boldsymbol{R}_{0}^{l}+\boldsymbol{R}_{0 \alpha}^{l l}, \\
\boldsymbol{R}_{0}^{l}+\boldsymbol{R}_{0 \beta}^{l m}=\boldsymbol{R}_{0}^{m}+\boldsymbol{R}_{0 \beta}^{m m} .
\end{gathered}
$$

These geometric relations define a change of variables:

$$
\left[\boldsymbol{R}, \boldsymbol{R}_{0}^{l}, \boldsymbol{R}_{0 \alpha}^{l l}, \boldsymbol{r}_{\alpha}^{l}\right] \rightleftharpoons\left[\boldsymbol{R}_{0}^{l}, \boldsymbol{R}_{0}^{m}, \boldsymbol{R}_{0 \beta}^{m m},-\boldsymbol{R}_{0 \beta}^{l m}\right]
$$


Notice that $\Theta_{0 \beta}^{l m}=\Theta_{\beta 0}^{m l}+\pi$, finally, we have

$$
\left(\boldsymbol{M}^{l m}\right)_{q r}=-\sum_{s=-\infty}^{\infty} \boldsymbol{T}_{q s}\left[\begin{array}{cc}
\mathcal{G}_{s r}^{c H} & 0 \\
0 & \mathcal{G}_{s r}^{c K}
\end{array}\right]
$$

where

$$
\begin{gathered}
\mathcal{G}_{s r}^{c H}=S_{r-s}^{c H}\left(k_{p} d_{y}, \sin \theta_{0}, \boldsymbol{R}_{0}^{l}, \boldsymbol{R}_{0}^{m}\right), \\
\mathcal{G}_{s r}^{c K}=(-1)^{s} S_{r-s}^{c K}\left(k_{p} d_{y}, \sin \theta_{0}, \boldsymbol{R}_{0}^{l}, \boldsymbol{R}_{0}^{m}\right) .
\end{gathered}
$$

The relationships listed in Eqs. (B.7) and (B.8) can also be treated from another perspective, and the change of variables is given below:

$$
\left[\boldsymbol{R}, \boldsymbol{R}_{0}^{l}, \boldsymbol{R}_{0 \alpha}^{l l}, \boldsymbol{r}_{\alpha}^{l}\right] \rightleftharpoons\left[\boldsymbol{R}_{0}^{m}, \boldsymbol{R}_{0}^{l},-\boldsymbol{R}_{0 \beta}^{m m}, \boldsymbol{R}_{0 \beta}^{l m}\right]
$$

After some algebraic operations, the results listed in Eqs. (B.11) and (B.12) are given in an equivalent form

$$
\begin{gathered}
\mathcal{G}_{s r}^{c H}=(-1)^{r-s} S_{r-s}^{c H}\left(k_{p} d_{y}, \sin \theta_{0}, \boldsymbol{R}_{0}^{m}, \boldsymbol{R}_{0}^{l}\right), \\
\mathcal{G}_{s r}^{c K}=(-1)^{r} S_{r-s}^{c K}\left(k_{p} d_{y}, \sin \theta_{0}, \boldsymbol{R}_{0}^{m}, \boldsymbol{R}_{0}^{l}\right) .
\end{gathered}
$$

In a similar way, the $\boldsymbol{M}$ matrix for a periodic lattice [see Eq. (42)] can be simplified by employing the lattice sums defined in Eqs. (A.5) and (A.6). The final form is

$$
\boldsymbol{M}_{q r}=\delta_{q r} \boldsymbol{I}-\sum_{s=-\infty}^{\infty} \boldsymbol{T}_{q s}\left[\begin{array}{cc}
(-1)^{r-s} S_{r-s}^{H}\left(k_{p}, \boldsymbol{K}\right) & 0 \\
0 & (-1)^{r} S_{r-s}^{K}\left(k_{p}, \boldsymbol{K}\right)
\end{array}\right]
$$

\section{Appendix C: Energy flux calculation}

For simplicity, the energy flux defined in Eq. (35) is rewritten as

$$
\left\langle F_{ \pm}\right\rangle= \pm \frac{\omega D}{2} \operatorname{Im}\left[\int_{y}^{y+d_{y}}\left(\eta_{1}-\eta_{2}\right) d y\right]
$$

where $\eta_{1}$ and $\eta_{2}$ are quantities with differential operations for total field $W$. As listed in Eq. (32), the total field is

$$
W=e^{i\left(k_{p x} x+k_{p y} y\right)}+\sum_{\sigma=-\infty}^{\infty} \zeta_{\sigma}^{H} e^{i\left[\kappa_{x}(\sigma) x+\kappa_{y}(\sigma) y\right]}+\sum_{\sigma=-\infty}^{\infty} \zeta_{\sigma}^{K} e^{-\left[\gamma_{x}(\sigma) x+\gamma_{y}(\sigma) y\right]} .
$$


Remember that $\boldsymbol{\kappa}_{\sigma}=k_{p}\left[\cos \tau_{1}(\sigma), \sin \tau_{1}(\sigma)\right]$ and $\boldsymbol{\gamma}_{\sigma}=k_{p}\left[\cos \tau_{2}(\sigma), \sin \tau_{2}(\sigma)\right]$. Differentiate $W$ with respect to the coordinate $x$, then we have

$$
\frac{\partial W}{\partial x}=i k_{p x} e^{i\left(k_{p x} x+k_{p y} y\right)}+\sum_{\sigma=-\infty}^{\infty} \zeta_{\sigma}^{H} i \kappa_{x}(\sigma) e^{i\left[\kappa_{x}(\sigma) x+\kappa_{y}(\sigma) y\right]}-\sum_{\sigma=-\infty}^{\infty} \zeta_{\sigma}^{K} \gamma_{x}(\sigma) e^{-\left[\gamma_{x}(\sigma) x+\gamma_{y}(\sigma) y\right]}
$$

Another term is

$$
\triangle W^{*}=-k_{p}^{2} e^{-i\left(k_{p x} x+k_{p y} y\right)}-k_{p}^{2} \sum_{\sigma=-\infty}^{\infty} \zeta_{\sigma}^{H *} e^{-i\left[\kappa_{x}^{*}(\sigma) x+\kappa_{y}^{*}(\sigma) y\right]}+k_{p}^{2} \sum_{\sigma=-\infty}^{\infty} \zeta_{\sigma}^{K *} e^{-\left[\gamma_{x}^{*}(\sigma) x+\gamma_{y}^{*}(\sigma) y\right]}
$$

and finally, the last term is

$$
\begin{aligned}
\frac{\partial}{\partial x}\left(\triangle W^{*}\right)=i k_{p}^{2} k_{p x} e^{-i\left(k_{p x} x+k_{p y} y\right)}+i k_{p}^{2} \sum_{\sigma=-\infty}^{\infty} \zeta_{\sigma}^{H *} \kappa_{x}^{*}(\sigma) e^{-i\left[\kappa_{x}^{*}(\sigma) x+\kappa_{y}^{*}(\sigma) y\right]} & \\
& -k_{p}^{2} \sum_{\sigma=-\infty}^{\infty} \zeta_{\sigma}^{K *} \gamma_{x}^{*}(\sigma) e^{-\left[\gamma_{x}^{*}(\sigma) x+\gamma_{y}^{*}(\sigma) y\right]}
\end{aligned}
$$

Let us proceed with the multiplication, after grouping we have

$$
\begin{aligned}
\eta_{1}=i k_{p}^{2} k_{p x} & +i k_{p}^{2} \sum_{\sigma=-\infty}^{\infty} \zeta_{\sigma}^{H *} \kappa_{x}^{*}(\sigma) e^{i\left[k_{p x}-\kappa_{x}^{*}(\sigma)\right] x} e^{i\left[k_{p y}-\kappa_{y}^{*}(\sigma)\right] y} \\
& -k_{p}^{2} \sum_{\sigma=-\infty}^{\infty} \zeta_{\sigma}^{K *} \gamma_{x}^{*}(\sigma) e^{i\left[k_{p x}+i \gamma_{x}^{*}(\sigma)\right] x} e^{i\left[k_{p y}+i \gamma_{y}^{*}(\sigma)\right] y} \\
& +i k_{p}^{2} k_{p x} \sum_{\sigma=-\infty}^{\infty} \zeta_{\sigma}^{H} e^{-i\left[k_{p x}-\kappa_{x}(\sigma)\right] x} e^{-i\left[k_{p y}-\kappa_{y}(\sigma)\right] y} \\
& +i k_{p}^{2} k_{p x} \sum_{\sigma=-\infty}^{\infty} \zeta_{\sigma}^{K} e^{-i\left[k_{p x}-i \gamma_{x}(\sigma)\right] x} e^{-i\left[k_{p y}-i \gamma_{y}(\sigma)\right] y} \\
& +i k_{p}^{2} \sum_{\sigma_{1}=-\infty}^{\infty} \sum_{\sigma_{2}=-\infty}^{\infty} \zeta_{\sigma_{1}}^{H} \zeta_{\sigma_{2}}^{H *} \kappa_{x}^{*}\left(\sigma_{2}\right) e^{i\left[\kappa_{x}\left(\sigma_{1}\right)-\kappa_{x}^{*}\left(\sigma_{2}\right)\right] x} e^{i\left[\kappa_{y}\left(\sigma_{1}\right)-\kappa_{y}^{*}\left(\sigma_{2}\right)\right] y} \\
& -k_{p}^{2} \sum_{\sigma_{1}=-\infty}^{\infty} \sum_{\sigma_{2}=-\infty}^{\infty} \zeta_{\sigma_{1}}^{H} \zeta_{\sigma_{2}}^{K *} \gamma_{x}^{*}\left(\sigma_{2}\right) e^{i\left[\kappa_{x}\left(\sigma_{1}\right)+i \gamma_{x}^{*}\left(\sigma_{2}\right)\right] x} e^{i\left[\kappa_{y}\left(\sigma_{1}\right)+i \gamma_{y}^{*}\left(\sigma_{2}\right)\right] y} \\
& +i k_{p}^{2} \sum_{\sigma_{1}=-\infty}^{\infty} \sum_{\sigma_{2}=-\infty}^{\infty} \zeta_{\sigma_{1}}^{K} \zeta_{\sigma_{2}}^{H *} \kappa_{x}^{*}\left(\sigma_{2}\right) e^{i\left[i \gamma_{x}\left(\sigma_{1}\right)-\kappa_{x}^{*}\left(\sigma_{2}\right)\right] x} e^{i\left[i \gamma_{y}\left(\sigma_{1}\right)-\kappa_{y}^{*}\left(\sigma_{2}\right)\right] y} \\
& -k_{p}^{2} \sum_{\sigma_{1}=-\infty}^{\infty} \sum_{\sigma_{2}=-\infty}^{\infty} \zeta_{\sigma_{1}}^{K} \zeta_{\sigma_{2}}^{K *} \gamma_{x}^{*}\left(\sigma_{2}\right) e^{-\left[\gamma_{x}\left(\sigma_{1}\right)+\gamma_{x}^{*}\left(\sigma_{2}\right)\right] x} e^{-\left[\gamma_{y}\left(\sigma_{1}\right)+\gamma_{y}^{*}\left(\sigma_{2}\right)\right] y},
\end{aligned}
$$


and

$$
\begin{aligned}
\eta_{2}=-i k_{p}^{2} k_{p x} & -i k_{p}^{2} \sum_{\sigma=-\infty}^{\infty} \zeta_{\sigma}^{H} \kappa_{x}(\sigma) e^{-i\left[k_{p x}-\kappa_{x}(\sigma)\right] x} e^{-i\left[k_{p y}-\kappa_{y}(\sigma)\right] y} \\
& +k_{p}^{2} \sum_{\sigma=-\infty}^{\infty} \zeta_{\sigma}^{K} \gamma_{x}(\sigma) e^{-i\left[k_{p x}-i \gamma_{x}(\sigma)\right] x} e^{-i\left[k_{p y}-i \gamma_{y}(\sigma)\right] y} \\
& -i k_{p}^{2} k_{p x} \sum_{\sigma=-\infty}^{\infty} \zeta_{\sigma}^{H *} e^{i\left[k_{p x}-\kappa_{x}^{*}(\sigma)\right] x} e^{i\left[k_{p y}-\kappa_{y}^{*}(\sigma)\right] y} \\
& +i k_{p}^{2} k_{p x} \sum_{\sigma=-\infty}^{\infty} \zeta_{\sigma}^{K *} e^{i\left[k_{p x}+i \gamma_{x}^{*}(\sigma)\right] x} e^{i\left[k_{p y}+i \gamma_{y}^{*}(\sigma)\right] y} \\
& -i k_{p}^{2} \sum_{\sigma_{1}=-\infty}^{\infty} \sum_{\sigma_{2}=-\infty}^{\infty} \zeta_{\sigma_{1}}^{H *} \zeta_{\sigma_{2}}^{H} \kappa_{x}\left(\sigma_{2}\right) e^{-i\left[\kappa_{x}^{*}\left(\sigma_{1}\right)-\kappa_{x}\left(\sigma_{2}\right)\right] x} e^{-i\left[\kappa_{y}^{*}\left(\sigma_{1}\right)-\kappa_{y}\left(\sigma_{2}\right)\right] y} \\
& +k_{p}^{2} \sum_{\sigma_{1}=-\infty}^{\infty} \sum_{\sigma_{2}=-\infty}^{\infty} \zeta_{\sigma_{1}}^{H *} \zeta_{\sigma_{2}}^{K} \gamma_{x}\left(\sigma_{2}\right) e^{-i\left[\kappa_{x}^{*}\left(\sigma_{1}\right)-i \gamma_{x}\left(\sigma_{2}\right)\right] x} e^{-i\left[\kappa_{y}^{*}\left(\sigma_{1}\right)-i \gamma_{y}\left(\sigma_{2}\right)\right] y} \\
& +i k_{p}^{2} \sum_{\sigma_{1}=-\infty}^{\infty} \sum_{\sigma_{2}=-\infty}^{\infty} \zeta_{\sigma_{1}}^{K *} \zeta_{\sigma_{2}}^{H} \kappa_{x}\left(\sigma_{2}\right) e^{i\left[i \gamma_{x}^{*}\left(\sigma_{1}\right)+\kappa_{x}\left(\sigma_{2}\right)\right] x} e^{i\left[i \gamma_{y}^{*}\left(\sigma_{1}\right)+\kappa_{y}\left(\sigma_{2}\right)\right] y} \\
& -k_{p}^{2} \sum_{\sigma_{1}=-\infty}^{\infty} \sum_{\sigma_{2}=-\infty}^{\infty} \zeta_{\sigma_{1}}^{K *} \zeta_{\sigma_{2}}^{K} \gamma_{x}\left(\sigma_{2}\right) e^{-\left[\gamma_{x}^{*}\left(\sigma_{1}\right)+\gamma_{x}\left(\sigma_{2}\right)\right] x} e^{-\left[\gamma_{y}^{*}\left(\sigma_{1}\right)+\gamma_{y}\left(\sigma_{2}\right)\right] y}
\end{aligned}
$$

From Appendix A, we know that

$$
\begin{gathered}
k_{p y}=k_{p} \sin \theta_{0}, \\
\kappa_{y}(\sigma)=\kappa_{y}^{*}(\sigma)=k_{p}\left(\sin \theta_{0}-\sigma \frac{2 \pi}{k_{p} d_{y}}\right), \\
\gamma_{y}(\sigma)=-\gamma_{y}^{*}(\sigma)=-i \kappa_{y}(\sigma),
\end{gathered}
$$

also

$$
\begin{gathered}
k_{p x}=k_{p} \cos \theta_{0}, \\
\kappa_{x}(0)=\kappa_{x}^{*}(0)= \pm k_{p x}, \\
\gamma_{x}(0)=\gamma_{x}^{*}(0)= \pm k_{p} \sqrt{1+\sin ^{2} \theta_{0}},
\end{gathered}
$$

where the positive and negative signs are used, respectively, in computing energy flux flowing along and opposite to the positive $x$-direction. With these relations, we obtain some vanishing integrals along the segment from $y$ to $y+d_{y}$ and these identities are listed below:

$$
\int_{y}^{y+d_{y}} e^{-i\left[k_{p y}-\kappa_{y}(\sigma)\right] y} d y=\int_{y}^{y+d_{y}} e^{-i \sigma \frac{2 \pi}{d y} y} d y=0, \quad \sigma \neq 0
$$




$$
\begin{aligned}
& \int_{y}^{y+d_{y}} e^{i\left[k_{p y}-\kappa_{y}^{*}(\sigma)\right] y} d y=\int_{y}^{y+d_{y}} e^{i\left[k_{p y}-\kappa_{y}(\sigma)\right] y} d y=0, \quad \sigma \neq 0 \\
& \int_{y}^{y+d_{y}} e^{-i\left[k_{p y}-i \gamma_{y}(\sigma)\right] y} d y=\int_{y}^{y+d_{y}} e^{-i\left[k_{p y}-\kappa_{y}(\sigma)\right] y} d y=0, \quad \sigma \neq 0 \\
& \int_{y}^{y+d_{y}} e^{i\left[k_{p y}+i \gamma_{y}^{*}(\sigma)\right] y} d y=\int_{y}^{y+d_{y}} e^{i\left[k_{p y}-\kappa_{y}(\sigma)\right] y} d y=0, \quad \sigma \neq 0 \\
& \int_{y}^{y+d_{y}} e^{i\left[\kappa_{y}\left(\sigma_{1}\right)-\kappa_{y}^{*}\left(\sigma_{2}\right)\right] y} d y=\int_{y}^{y+d_{y}} e^{i\left[\left(\sigma_{1}-\sigma_{2}\right) \frac{2 \pi}{d y}\right] y} d y=0, \quad \sigma_{1} \neq \sigma_{2} \\
& \int_{y}^{y+d_{y}} e^{-i\left[\kappa_{y}^{*}\left(\sigma_{1}\right)-\kappa_{y}\left(\sigma_{2}\right)\right] y} d y=\int_{y}^{y+d_{y}} e^{-i\left[\kappa_{y}\left(\sigma_{1}\right)-\kappa_{y}\left(\sigma_{2}\right)\right] y} d y=0, \quad \sigma_{1} \neq \sigma_{2} \\
& \int_{y}^{y+d_{y}} e^{i\left[\kappa_{y}\left(\sigma_{1}\right)+i \gamma_{y}^{*}\left(\sigma_{2}\right)\right] y} d y=\int_{y}^{y+d_{y}} e^{i\left[\kappa_{y}\left(\sigma_{1}\right)-\kappa_{y}^{*}\left(\sigma_{2}\right)\right] y} d y=0, \quad \sigma_{1} \neq \sigma_{2} \\
& \int_{y}^{y+d_{y}} e^{-i\left[\kappa_{y}^{*}\left(\sigma_{1}\right)-i \gamma_{y}\left(\sigma_{2}\right)\right] y} d y=\int_{y}^{y+d_{y}} e^{-i\left[\kappa_{y}\left(\sigma_{1}\right)-\kappa_{y}\left(\sigma_{2}\right)\right] y} d y=0, \quad \sigma_{1} \neq \sigma_{2} \\
& \int_{y}^{y+d_{y}} e^{i\left[i \gamma_{y}\left(\sigma_{1}\right)-\kappa_{y}^{*}\left(\sigma_{2}\right)\right] y} d y=\int_{y}^{y+d_{y}} e^{i\left[\kappa_{y}\left(\sigma_{1}\right)-\kappa_{y}^{*}\left(\sigma_{2}\right)\right] y} d y=0, \quad \sigma_{1} \neq \sigma_{2} \\
& \int_{y}^{y+d_{y}} e^{i\left[i \gamma_{y}^{*}\left(\sigma_{1}\right)+\kappa_{y}\left(\sigma_{2}\right)\right] y} d y=\int_{y}^{y+d_{y}} e^{i\left[-\kappa_{y}\left(\sigma_{1}\right)+\kappa_{y}\left(\sigma_{2}\right)\right] y} d y=0, \quad \sigma_{1} \neq \sigma_{2} \\
& \int_{y}^{y+d_{y}} e^{-\left[\gamma_{y}\left(\sigma_{1}\right)+\gamma_{y}^{*}\left(\sigma_{2}\right)\right] y} d y=\int_{y}^{y+d_{y}} e^{i\left[\kappa_{y}\left(\sigma_{1}\right)-\kappa_{y}^{*}\left(\sigma_{2}\right)\right] y} d y=0, \quad \sigma_{1} \neq \sigma_{2} \\
& \int_{y}^{y+d_{y}} e^{-\left[\gamma_{y}^{*}\left(\sigma_{1}\right)+\gamma_{y}\left(\sigma_{2}\right)\right] y} d y=\int_{y}^{y+d_{y}} e^{-i\left[-\kappa_{y}\left(\sigma_{1}\right)+\kappa_{y}\left(\sigma_{2}\right)\right] y} d y=0, \quad \sigma_{1} \neq \sigma_{2}
\end{aligned}
$$

Combining the previous equations, we have the following formulas after tedious algebraic operations

$$
\begin{aligned}
\frac{1}{d_{y}} \int_{y}^{y+d_{y}} \eta_{1} d y= & i k_{p}^{2} k_{p x}+i k_{p}^{2} k_{p x} \zeta_{0}^{H}+i k_{p}^{2} \kappa_{x}^{*}(0) \zeta_{0}^{H *} \\
& -k_{p}^{2} \gamma_{x}^{*}(0) \zeta_{0}^{K *} e^{i\left[k_{p x}+i \gamma_{x}^{*}(0)\right] x}+i k_{p}^{2} k_{p x} \zeta_{0}^{K} e^{-i\left[k_{p x}-i \gamma_{x}(0)\right] x} \\
& +i k_{p}^{2} \sum_{\sigma=-\infty}^{\infty} \kappa_{x}^{*}(\sigma) \zeta_{\sigma}^{H} \zeta_{\sigma}^{H *}-k_{p}^{2} \sum_{\sigma=-\infty}^{\infty} \gamma_{x}^{*}(\sigma) \zeta_{\sigma}^{H} \zeta_{\sigma}^{K *} e^{i\left[\kappa_{x}(\sigma)+i \gamma_{x}^{*}(\sigma)\right] x} \\
& +i k_{p}^{2} \sum_{\sigma=-\infty}^{\infty} \kappa_{x}^{*}(\sigma) \zeta_{\sigma}^{K} \zeta_{\sigma}^{H *} e^{i\left[i \gamma_{x}(\sigma)-\kappa_{x}^{*}(\sigma)\right] x}-k_{p}^{2} \sum_{\sigma=-\infty}^{\infty} \gamma_{x}^{*}(\sigma) \zeta_{\sigma}^{K} \zeta_{\sigma}^{K *} e^{-\left[\gamma_{x}(\sigma)+\gamma_{x}^{*}(\sigma)\right] x}
\end{aligned}
$$


and

$$
\begin{aligned}
\frac{1}{d_{y}} \int_{y}^{y+d_{y}} \eta_{2} d y= & -i k_{p}^{2} k_{p x}-i k_{p}^{2} k_{p x} \zeta_{0}^{H *}-i k_{p}^{2} \kappa_{x}(0) \zeta_{0}^{H} \\
& +k_{p}^{2} \gamma_{x}(0) \zeta_{0}^{K} e^{-i\left[k_{p x}-i \gamma_{x}(0)\right] x}+i k_{p}^{2} k_{p x} \zeta_{0}^{K *} e^{i\left[k_{p x}+i \gamma_{x}^{*}(0)\right] x} \\
& -i k_{p}^{2} \sum_{\sigma=-\infty}^{\infty} \kappa_{x}(\sigma) \zeta_{\sigma}^{H *} \zeta_{\sigma}^{H}+k_{p}^{2} \sum_{\sigma=-\infty}^{\infty} \gamma_{x}(\sigma) \zeta_{\sigma}^{H *} \zeta_{\sigma}^{K} e^{-i\left[\kappa_{x}^{*}(\sigma)-i \gamma_{x}(\sigma)\right] x} \\
& +i k_{p}^{2} \sum_{\sigma=-\infty}^{\infty} \kappa_{x}(\sigma) \zeta_{\sigma}^{K *} \zeta_{\sigma}^{H} e^{i\left[i \gamma_{x}^{*}(\sigma)+\kappa_{x}(\sigma)\right] x}-k_{p}^{2} \sum_{\sigma=-\infty}^{\infty} \gamma_{x}(\sigma) \zeta_{\sigma}^{K *} \zeta_{\sigma}^{K} e^{-\left[\gamma_{x}^{*}(\sigma)+\gamma_{x}(\sigma)\right] x}
\end{aligned}
$$

Substituting Eqs. (C.26) and (C.27) into Eq. (C.1) and using the properties of complex operation, finally, we obtain the following result

$$
\left\langle F_{ \pm}\right\rangle= \pm \omega d_{y} D k_{p}^{3}\left\{\cos \theta_{0}+(1 \pm 1) \operatorname{Re}\left[\zeta_{0}^{H}\right] \cos \theta_{0} \pm \sum_{\cos \tau_{1}(\sigma) \in \mathbb{R}}\left|\zeta_{\sigma}^{H}\right|^{2}\left|\cos \tau_{1}(\sigma)\right|\right\} .
$$

\section{Appendix D: Modeling 2-beam resonators as spring-mass oscillator}

As observed in Fig. 3, the 2-beam resonators show several resonance modes in the frequency range of interest and a low frequency band gap is opened by the flexural resonance. The aim of this appendix is to study the resonant frequency by means of the spring-mass model, as shown in Figure D.1. When the flexural resonance occurs, the bridges behave like an extension spring with effective stiffness $K_{e}$ and the inner plate serves as the mass $M$. The resonant frequency is determined by the following formula

$$
f=\frac{1}{2 \pi} \sqrt{\frac{K_{e}}{M}}
$$

The main task of the following paragraph is to determine the effective stiffness of the beams. For convenience, a Cartesian coordinates system $O-x y z$ is built at the center of the resonator. The $x$ - and $y$-axis determine the plane of the thin plate and the $z$-axis points to the out-ofplane direction.

It is assumed that a harmonic force with amplitude $F$ is exerted at the center of the inner plate. Both the bridges and inner plate are considered as beams under the action of bending moments resulting from the force. According to the beam theory, ${ }^{29}$ we obtain the equations of motion for the bridges and inner plate, respectively, as

$$
E \mathrm{I} \frac{d^{2} w_{b}}{d x^{2}}=\frac{F}{2}\left(x-R_{1}\right), \quad x \in\left[R_{1}, R_{2}\right]
$$


(a)

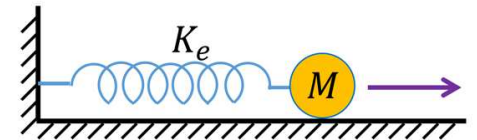

(b)

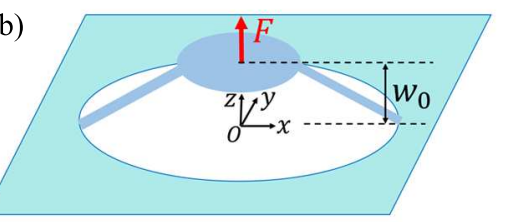

FIG. D.1. (color on line) (a) Scheme of a typical spring-mass resonator. (b) Scheme of a 2-beam resonator, its analogue in a thin plate.

and

$$
E \mathrm{I} \frac{d^{2} w_{p}}{d x^{2}}=-\frac{F R_{1}}{2}, \quad x \in\left[0, R_{1}\right]
$$

where $w_{b}$ and $w_{p}$ are the out-of-plane displacements for the bridges and inner plate, respectively. Notice that the inner plate is treated as a beam of variable cross-section and the second moment of area should be replaced by $\mathrm{I}=h^{3} \sqrt{R_{1}^{2}-x^{2}} / 6$. The previous equations can be solved by considering the boundary conditions; the end of the bridge connected to the background plate is considered as clamped, while at the other end, the displacement and slope on both sides should be continuous. The expressions are here listed:

$$
\begin{gathered}
\left.w_{b}\right|_{x=R_{2}}=0 \text { and }\left.\frac{d w_{b}}{d x}\right|_{x=R_{2}}=0, \\
\left.w_{b}\right|_{x=R_{1}}=\left.w_{p}\right|_{x=R_{1}} \text { and }\left.\frac{d w_{b}}{d x}\right|_{x=R_{1}}=\left.\frac{d w_{p}}{d x}\right|_{x=R_{1}} .
\end{gathered}
$$

Combining Eqs. (D.2)-(D.5), the maximum displacement of the inner plate is obtained as

$$
w_{0}=\frac{F\left(R_{2}-R_{1}\right)^{2}\left(R_{1}+2 R_{2}\right)}{E b h^{3}}-\frac{3 F R_{1}^{2}}{E h^{3}} .
$$

Finally, the resonant frequency is available by substituting the following effective parameters into Eq. (D.1)

$$
K_{e}=\frac{F}{w_{0}} \quad \text { and } \quad M=\pi \rho h R_{1}^{2} .
$$

\section{REFERENCES}

${ }^{1}$ T.-T. Wu, J.-C. Hsu, and J.-H. Sun, IEEE transactions on ultrasonics, ferroelectrics, and frequency control 58, 2146 (2011). 
${ }^{2}$ R. Zhu, X. Liu, G. Hu, F. Yuan, and G. Huang, International Journal of Smart and Nano Materials 6, 14 (2015).

${ }^{3}$ D. Torrent, Y. Pennec, and B. Djafari-Rouhani, Phys. Rev. B 90, 104110 (2014).

${ }^{4}$ R. Zhu, X. Liu, G. Hu, C. Sun, and G. Huang, Nature communications 5, 5510 (2014).

${ }^{5}$ H. Lee, J. H. Oh, H. M. Seung, S. H. Cho, and Y. Y. Kim, Scientific reports 6, 24026 (2016).

${ }^{6}$ R. Zhu, Y. Chen, Y. Wang, G. Hu, and G. Huang, J. Acous. Soc. Am. 139, 3303 (2016).

${ }^{7}$ S.-C. S. Lin, T. J. Huang, J.-H. Sun, and T.-T. Wu, Phys. Rev. B 79, 094302 (2009).

${ }^{8}$ A. Climente, D. Torrent, and J. Sánchez-Dehesa, Applied Physics Letters 97, 104103 (2010).

${ }^{9}$ T.-T. Wu, Y.-T. Chen, J.-H. Sun, S.-C. S. Lin, and T. J. Huang, Appl. Phys. Lett. 98, 171911 (2011).

${ }^{10}$ Y. Jin, D. Torrent, Y. Pennec, Y. Pan, and B. Djafari-Rouhani, Scientific reports 6, 24437 (2016).

${ }^{11}$ M. Farhat, S. Guenneau, S. Enoch, A. B. Movchan, and G. G. Petursson, Appl. Phys. Lett. 96, 081909 (2010).

${ }^{12}$ X. Liu, G. Hu, G. Huang, and C. Sun, Applied physics letters 98, 251907 (2011).

${ }^{13}$ M. Farhat, S. Enoch, and S. Guenneau, EPL (Europhysics Letters) 107, 44002 (2014).

${ }^{14}$ S. Tol, F. Degertekin, and A. Erturk, Applied Physics Letters 109, 063902 (2016).

${ }^{15}$ A. Darabi and M. J. Leamy, Smart Materials and Structures 26 (2017).

${ }^{16}$ J. B. Pendry, Physical review letters 85, 3966 (2000).

${ }^{17}$ P. A. Deymier, Acoustic metamaterials and phononic crystals, Vol. 173 (Springer Science \& Business Media, 2013).

${ }^{18}$ M. Badreddine Assouar, M. Senesi, M. Oudich, M. Ruzzene, and Z. Hou, Applied Physics Letters 101, 173505 (2012).

${ }^{19}$ J.-C. Hsu and T.-T. Wu, Applied physics letters 90, 201904 (2007).

${ }^{20}$ W. Xiao, G. Zeng, and Y. Cheng, Applied Acoustics 69, 255 (2008).

${ }^{21}$ M. Oudich, Y. Li, B. M. Assouar, and Z. Hou, New Journal of Physics 12, 083049 (2010).

${ }^{22}$ M. Oudich, M. B. Assouar, and Z. Hou, Applied Physics Letters 97, 193503 (2010).

${ }^{23}$ M. Oudich, M. Senesi, M. B. Assouar, M. Ruzenne, J.-H. Sun, B. Vincent, Z. Hou, and T.-T. Wu, Physical Review B 84, 165136 (2011).

${ }^{24}$ Y. Xiao, J. Wen, and X. Wen, Journal of Physics D: Applied Physics 45, 195401 (2012). 
${ }^{25}$ E. Andreassen, K. Manktelow, and M. Ruzzene, Journal of Sound and Vibration 335, 187 (2015).

${ }^{26}$ A. Climente, P. Gao, L. Wu, and J. Sánchez-Dehesa, ArXiv [physics.class-ph] , 1710.04610 (2017).

${ }^{27}$ W. Parnell and P. Martin, Wave Motion 48, 161 (2011).

${ }^{28}$ L.-W. Cai and S. A. Hambric, Journal of Vibration and Acoustics 138, 011009 (2016).

${ }^{29}$ K. F. Graff, Wave motion in elastic solids (Courier Corporation, 2012).

${ }^{30} \mathrm{G}$. N. Watson, A treatise on the theory of Bessel functions (Cambridge university press, 1995).

${ }^{31}$ A. Norris and C. Vemula, Journal of sound and vibration 181, 115 (1995).

${ }^{32}$ U. Fano, JOSA 31, 213 (1941).

${ }^{33} \mathrm{~V}$. Twersky, IRE Transactions on antennas and propagation 10, 737 (1962).

${ }^{34}$ A. Movchan, N. Movchan, and R. McPhedran, in Proceedings of the Royal Society of London A: Mathematical, Physical and Engineering Sciences, Vol. 463 (The Royal Society, 2007) pp. 2505-2518.

${ }^{35}$ J. V. Sánchez-Pérez, D. Caballero, R. Mártinez-Sala, C. Rubio, J. Sánchez-Dehesa, F. Meseguer, J. Llinares, and F. Gálvez, Phys. Rev. Lett. 80, 5325 (1998).

${ }^{36}$ F.-L. Hsiao, A. Khelif, H. Moubchir, A. Choujaa, C.-C. Chen, and V. Laude, Journal of applied physics 101, 044903 (2007).

${ }^{37}$ F. Cervera, L. Sanchis, J. Sánchez-Pérez, R. Martinez-Sala, C. Rubio, F. Meseguer, C. López, D. Caballero, and J. Sánchez-Dehesa, Physical review letters 88, 023902 (2001).

${ }^{38} \mathrm{D}$. T. Martí, Towards the full control of sound with sonic crystals and acoustic metamaterials, Ph.D. thesis, Universitat Politècnica de València (2008).

${ }^{39} \mathrm{M}$. Abramowitz and I. A. Stegun, Handbook of mathematical functions: with formulas, graphs, and mathematical tables, Vol. 55 (Courier Corporation, 1964). 